\title{
Frequency-dependent viscosity of xenon near the critical point
}

\author{
Robert F. Berg, Michael R. Moldover, \\ Physical and Chemical Properties Division \\ National Institute of Standards and Technology \\ Gaithersburg, MD 20899-8380 USA \\ and Gregory A. Zimmerli \\ NYMA Incorporated, \\ National Center for Microgravity Research \\ c/o NASA Lewis Research Center, MS 110-3 \\ Cleveland, OH 44135 \\ 20 January 1999
}




\begin{abstract}
We used a novel, overdamped oscillator aboard the Space Shuttle to measure the viscosity $\eta$ of xenon near its critical density $\rho_{c}$ and temperature $T_{c}$. In microgravity, useful data were obtained within $0.1 \mathrm{mK}$ of $T_{c}$, corresponding to a reduced temperature $t=\left(T-T_{c}\right) / T_{c}=$ $3 \times 10^{-7}$. The data extend two decades closer to $T_{c}$ than the best ground measurements, and they directly reveal the expected power-law behavior $\eta \propto t^{-\nu z_{\eta}}$. Here $\nu$ is the correlation length exponent, and our result for the small viscosity exponent is $z_{\eta}=0.0690 \pm 0.0006$. (All uncertainties are one standard uncertainty.) Our value for $z_{\eta}$ depends only weakly on the form of the viscosity crossover function, and it agrees with the value $0.067 \pm 0.002$ obtained from a recent two-loop perturbation expansion [H. Hao, R.A. Ferrell, and J.K. Bhattacharjee, preprint (1997)]. The measurements spanned the frequency range $2 \mathrm{~Hz} \leq f \leq 12 \mathrm{~Hz}$ and revealed viscoelasticity when $t \leq 10^{-5}$, further from $T_{c}$ than predicted. The viscoelasticity scales as $A f \tau$, where $\tau$ is the fluctuation-decay time. The fitted value of the viscoelastic timescale parameter $A$ is $2.0 \pm 0.3$ times the result of a one-loop perturbation calculation. Near $T_{c}$, the xenon's calculated time constant for thermal diffusion exceeded days. Nevertheless, the viscosity results were independent of the xenon's temperature history, indicating that the density was kept near $\rho_{c}$ by judicious choices of the temperature vs. time program. Deliberately bad choices led to large density inhomogeneities. At $t>10^{-5}$, the xenon approached equilibrium much faster than expected, suggesting that convection driven by microgravity and by electric fields slowly stirred the sample.
\end{abstract}

PACS index categories: $51.20 .+\mathrm{d} ; 05.70 \mathrm{Jk} ; 64.60 . \mathrm{Ht} ; 83.50 . \mathrm{Fc} ; 83.85 . \mathrm{Jn}$ 


\section{Introduction}

As the liquid-vapor critical point is approached, the shear viscosity $\eta(\xi)$ measured in the limit of zero frequency diverges as $\xi^{z_{\eta}}$, where $\xi$ is the correlation length, which itself diverges on the critical isochore as $t^{-\nu}$. (Here, $t=\left(T-T_{c}\right), T_{c}$ is the critical temperature, and $\nu=0.630$.) The viscosity exponent $z_{\eta}$ is central to the theory of dynamic critical phenomena [1]; the quantity $3+z_{\eta}$ is called the "dynamic critical exponent" and it characterizes transport phenomena in all near-critical fluids with a scalar order parameter. Because the exponent $z_{\eta}=0.069$ is so small, it is very difficult to measure accurately on Earth in a pure fluid such as xenon. Far from the critical point, the separation of the viscosity's divergent critical contribution from the analytic background contribution is uncertain because the critical contribution is a small fraction of the total and because the separation depends sensitively upon the "crossover function" $H(\xi)$, which is known only approximately. Close to the critical point, the very compressible xenon stratifies in the Earth's gravity and the divergence of the viscosity is blunted in a manner that depends upon the height of the viscometer. As illustrated by Figure 1, stratification visibly blunted the divergence of the viscosity of xenon near $t_{\min }=3 \times 10^{-5}$, even when the measurements were made using a high- $Q$ oscillator only $0.7 \mathrm{~mm}$ high. The arguments of Ref. [2] show that $t_{\min }$ scales with the height $h$ and the acceleration of gravity $g$ as $(g h)^{0.64}$. Thus, a significant reduction of $t_{\min }$ requires either a viscometer with $h<<0.7 \mathrm{~mm}$ on Earth or the use of microgravity.

Here we report results obtained with a novel viscometer that we integrated into the "Critical Viscosity of Xenon" (CVX) experiment package and operated aboard the Space Shuttle Mission STS-85. In the Shuttle's microgravity environment (approximately $1 \times 10^{-5}$ $\mathrm{m} \cdot \mathrm{s}^{-2}$ ), CVX obtained useful viscosity data at $t_{\text {tnin }}=3 \times 10^{-7}$, two decades in $t$ closer to $T_{c}$ than the best ground-based measurements. Indeed, the present data are useful closer to $T_{c}$ than the data from any prior studies of liquid-vapor critical points, including those conducted in microgravity (e.g. $[3,4,5][6,7])$. The CVX data yielded a more accurate value of $z_{\eta}$ than previous work, and it also produced the first accurate measurements of the frequency dependence of viscosity near any critical point. 
The CVX viscometer was a torsion oscillator that was driven at frequencies in the range $2 \mathrm{~Hz} \leq f \leq 12 \mathrm{~Hz}$. Both the in-phase and the quadrature components of the oscillator's response to the driving torque were measured. This permitted us to separately deduce the real and the imaginary components of the viscosity, $\operatorname{Re}(\eta)$ and $\operatorname{Im}(\eta)$. Both components are required to fully test theory. The imaginary component is the elastic part of the response to shear stress that is ordinarily studied either at much higher frequencies or in complicated fluids such as polymer melts. In near-critical fluids, $\operatorname{Im}(\eta) \neq 0$ when $f \tau \gtrsim 1$, where $f$ is the frequency of the measurement and $\tau$ is the fluctuation-decay time

$$
\tau=\frac{6 \pi \eta \xi^{3}}{k_{B} T_{c}}=\tau_{0} t^{-\nu\left(3+z_{\eta}\right)}
$$

where $k_{B}$ is Boltzmann's constant. At the very low frequencies used by CVX, the viscoelastic behavior of xenon was evident when $t=10^{-5}$ and it was dominant when $t=10^{-6}$. (See Figure 2.)

The theoretical model used to analyze the CVX data combined the scaling function $S(z)$ for near-critical viscoelasticity from Ref. [8], the crossover function $H(\xi)$ from Ref. [9], and the background viscosity $\eta_{0}$ from Appendix $\mathrm{C}$ of Ref. [10] to obtain the prediction

$$
\eta(\xi, f)=\eta_{0} \exp \left[z_{\eta} H(\xi)\right][S(A z)]^{-z_{\eta} /\left(3+z_{\eta}\right)} .
$$

In Eq.(1.2), the scaled frequency is defined by $z=-i \pi f \tau$, and we introduced the parameter $A$ into the argument of the scaling function $S(z)$ to obtain agreement with our data. Accurate measurements of the scaling function require accurate measurements of $\eta(\xi, f)$ in the region where is large and where $z$ is varied through a large range from $z<<1$ to $z>>1$. Measurements prior to CVX were unable to achieve these conditions [11, 12, 13, 14].

The CVX viscosity data at $2 \mathrm{~Hz}, 3 \mathrm{~Hz}, 5 \mathrm{~Hz}, 8 \mathrm{~Hz}$, and $12 \mathrm{~Hz}$ were used to determine five parameters. Two are the "universal" parameters $z_{\eta}$ and $A$; two are the wave vectors $q_{C}$ and $q_{D}$ that occur in the crossover function for xenon; and one is the value of $T_{c}$ on CVX's temperature scale. When all of the data within the range $10^{-6}<t<10^{-4}$ were fitted for these parameters, we obtained $z_{\eta}=0.0690 \pm 0.0006, q_{C}=0.051 \pm 0.007$, and $q_{D}=0.16 \pm 0.05$. (The uncertainties indicated thronghout this report are one standard uncertainty and they 
As discussed in Section 5.3, the CVX data determined the product $A \tau_{0}=2.31 \pm 0.06 \mathrm{ps}$ with a relative uncertainty of 0.03 . The relative uncertainty of $A$ is larger $(0.15)$ because of the uncertainty of the correlation length amplitude $\xi_{0}$ that propagated into $\tau_{0}$ and then into $A \tau_{0}$. The uncertainty $A$ could be reduced by a factor of five if the uncertainty of $\xi_{0}$ were reduced, perhaps by additional measurements on Earth.

The present research was preceded by viscosity studies on Earth of xenon and carbon dioxide near their liquid-vapor critical points [10] and binary liquid mixtures near their consolute points [14]. The latter belong to the same dynamic universality class as pure fluids but are less influenced by gravity. We now identify the ways that CVX complements the previous research.

For tests of theory, i.e. Eq.(1.2), a simple pure fluid such as xenon has three advantages in comparison with binary liquid mixtures near consolute points. First, the dependences of the noncritical viscosity $\eta_{0}(\rho, T)$ on density $\rho$ and temperature $T$ are weak compared with the dependencies of $\eta_{0}(x, T)$ on mole fraction $x$ and temperature for binary liquids. For pure fluids, the temperature dependence is indistinguishable from the well-understood dilute gas behavior $\eta_{0}(0, T)[19]$. This means that $\eta_{0}(\rho, T)$ has a temperature dependence that is weaker than for binary liquids, and its use in fits to Eq.(1.2) adds no free parameters. Second, the fluid-dependent parameter $q_{C}$ that appears in the crossover function $H(\xi)[9]$ is known for xenon; it has not been determined for any binary liquid. (The other parameter in $H(\xi)$ is the effective cutoff wave vector $q_{D}$ that is used in a mode coupling integral over momentum space. It is a free parameter related to the amplitude of the viscosity divergence.) Third, current technology allows the conditions of low frequency and shear rate to be met more easily with pure fluids than with liquid mixtures because the decay time $\tau$ is at least 100 times faster in pure fluids than in mixtures at the same reduced temperature.

Major divisions of the remainder of this manuscript are titled: Apparatus, Temperature timeline, Data reduction, and Results. Appendices deal with: Tabulated viscosity data, Electrostriction, Frequency-dependent scaling function $S(z)$, and Estimation of $q_{C}$. 


\section{Apparatus}

The flight apparatus consisted of the cell holding the xenon sample and the oscillator, the surrounding thermostat, and the electronics, all contained in two flight cannisters. Here we provide an overview. Additional details can be found in References [17] and [18].

\subsection{Oscillator and sample cell}

The heart of the CVX viscometer, shown in Figure 5, was an oscillator contained in a thickwalled copper sample cell. The oscillator was an $8 \times 19 \mathrm{~mm}$ rectangle of screen that was cut out of a larger piece of nickel screen. The screen consisted of $0.03-\mathrm{mm}$ wide wires formed by electrodeposition (Buckbee-Mears [20]) and spaced $0.85 \mathrm{~mm}$ apart. When the screen was cut, two wires were left extending from the edges of the rectangle. They served as torsion wires and were soldered to a stiff yoke that was centered between four electrodes parallel to the screen. We chose the oscillator's dimensions so that it could be assembled by hand and so that it would be sensitive to changes of viscosity at low frequency. The oscillator's mass was approximately $1 \mathrm{mg}$.

Four electrodes were used to apply torque to the oscillator. To do so, diagonally opposite pairs of electrodes were charged to different voltages while the screen remained grounded. The electrodes were $10 \times 12 \mathrm{~mm}$ rectangles of $0.13 \mathrm{~mm}$ thick brass sheet soldered to $1 \mathrm{~mm}$ diameter wires that acted as both mechanical supports and as electrical leads. The electrodes lay in two planes located approximately $4 \mathrm{~mm}$ above and below the plane of the oscillator. The screen's yoke and the four electrodes were supported by feedthroughs in the brass end plate. The complete assembly was inspected for dust and then placed into the sample cell.

The sample cell had an outer diameter that was $38 \mathrm{~mm}$, and an inner, cylindrical space was $38 \mathrm{~mm}$ long and $19 \mathrm{~mm}$ in diameter. One end of the cell was sealed by a sapphire window that had been coated with tin oxide to eliminate static charges. The other end was sealed by a brass plate containing five electrical feedthroughs connected to the oscillator and the four surrounding electrodes. The two sealing gaskets were made of indium-coated copper wire. The seals and feedthroughs were tested for leaks with a mass spectrometcr 
leak detector while the cell was pressurized with helium at $12 \mathrm{MPa}$. The cell was heated to $100^{\circ} \mathrm{C}$, pumped for four hours to remove volatile contaminants; then it was cooled to room temperature and isolated from the vacuum pump. The oscillator's quality factor $Q$ was monitored during the next four days. The time dependence of $Q$ indicated that outgassing was negligible.

The torsion oscillator's resonance frequency $f_{0}$, quality factor $Q$, and elastic aftereffect were measured in vacuum as functions of temperature. These measurements were used later in a model of the oscillator's anelasticity to predict the frequency and temperature dependence of the oscillator's spring constant and its internal losses [21] at all frequencies. Near $T_{c}, f_{0} \simeq 11 \mathrm{~Hz}$ and $Q \simeq 1000$. See Figure 6 . No other mode was detected at frequencies between $0 \mathrm{~Hz}$ and $100 \mathrm{~Hz}$.

\subsection{Loading xenon at critical density}

In order to obtain meaningful viscosity data close to $T_{c}$, the density of the xenon near the oscillator had to be within a few percent of $\rho_{c}$. To attain this we controlled both the average density and the density gradients. The average density was determined when the cell was loaded.

After characterization of the oscillator in vacuum, the sample cell was filled with xenon (Matheson 99.995\%) through a copper fill line. To adjust the loading, the cell was immersed and held horizontally in a thermostatted fish tank at a temperature less than $2 \mathrm{mK}$ below $T_{c}$. Xenon was added or removed to bring the liquid-vapor meniscus to the cell's midplane. The fill line was crimped, cut, and sealed by soldering. Epoxy was poured around the electrical feedthroughs as a precaution against leaks. The cell was weighed one year before and one year after the flight. The difference between the weighings indicated that less than $0.02 \%$ of the sample was lost during this period.

We observed the height of the meniscus when the sample was in equilibrium a few $\mathrm{mK}$ below $T_{c}$. The inferred average density was $(1-0.0015 \pm 0.0017) \rho_{c}$. This difference from $\rho_{c}$

decreased the correlation length. At the lower bound of the fitting range, $t=1 \times 10^{-6}$, the 
corresponding decrease of the viscosity at $0 \mathrm{~Hz}$ was $\left(0.10_{-0.00}^{+0.36}\right) \%$.

We measured $T_{c}$ by recording the temperatures at which the meniscus appeared and disappeared while the cell was immersed in the fish tank. The thermometer was a platinum resistance thermometer immersed near the cell. Measurements made three years and one year before the flight fell in the range $T_{c}=289.736 \pm 0.006 \mathrm{~K}$.

The xenon lowered the oscillator's $Q$ from 1000 to less than 1 . This overdamping made the viscometer insensitive to vibrations associated with normal Shuttle operations and it led to other advantages. The overdamped oscillator was sensitive to viscosity changes in the range 2 to $12 \mathrm{~Hz}$. This allowed the oscillator to be calibrated by exploiting a hydrodynamic similarity. The data at multiple frequencies provided a powerful check on the viscometer's accuracy, and they helped reveal the viscoelasticity of xenon near $T_{c}$. Finally, the overdamping made the oscillator rugged enough to survive the strong accelerations associated with launch and touchdown.

\subsection{Thermostat}

Maintaining sample homogeneity near $T_{c}$ required that temperature differences within the sample be small. The CVX thermostat achieved such small temperature differences. It consisted of three concentric aluminum shells surrounding the thick-walled copper sample cell. The cylindrical shells and their end caps were made from 6-mm-thick aluminum with a radial gap of $13 \mathrm{~mm}$ between shells. The large radial gap and stiff, glass-filled polycarbonate spacers between shells made the design mechanically robust and insensitive to errors of design and construction. The 38-mm separation between end caps allowed easy installation of the cell and its wiring. The weak coupling between shells that resulted from the large gaps increased the thermostat's response time to more than one hour: However, this was acceptable because the thermostat's response time was less than the sample's internal response time near the critical point. The thermostat's construction and operation are described in more detail elsewhere $[18,17,22]$.

The performance of the thermostat was verified by using a semiconductor thermopile to 
measure the temperature difference imposed along a thin-walled steel sample cell both while controlling the thermostat at constant temperature and while ramping it at $10 \mu \mathrm{K} \cdot \mathrm{s}^{-1}$ [22]. Extrapolating the results to CVX indicated that, while ramping at the slowest rate of -0.05 $\mu \mathrm{K} \cdot \mathrm{s}^{-1}$, the temperature difference along the thick-walled copper cell was only $-0.11 \mu \mathrm{K}$. We added half of this value to the difference caused by the thermistor's power to obtain a maximum difference of $0.11 \mu \mathrm{K}$ of the cell's wall temperature from its average value. This corresponded to a density difference of $0.003 \rho_{\mathrm{c}}$ at $t=1 \times 10^{-6}$, which was acceptable.

\subsection{Flight cannisters}

The CVX flight package consisted of two sealed 0.8-m-tall aluminum "Hitchhiker" cannisters. The "Experiment" cannister, shown in Figure 7, contained the thermostat and the more sensitive analog electronics. It also contained electrical batteries that were used to maintain the thermostat's temperature above $T_{c}$ during descent when no power was available from the Shuttle. Keeping the sample above $T_{c}$ during descent prevented the formation of liquid whose sloshing could have damaged the oscillator. The "Avionics" cannister contained power conditioners, an accelerometer, and the data acquisition and control electronics. It also contained the four computers dedicated to the tasks of viscometry, temperature control, accelerometry, and communications.

The cannisters were mounted in the Space Shuttle's open payload bay, where the external heat load varied greatly depending on whether the bay was oriented toward deep space, the Earth, or the Sun. These heat load variations changed the Experiment cannister's interior temperature, which in turn affected the gain of the viscometry electronics. To minimize these temperature changes, the sides of the cannister were thermally isolated and the mass of the cannister's lid was increased to $45 \mathrm{~kg}$. A heater attached to the underside of the lid provided additional temperature control. Between mission days 1 and 10, the Experiment cannister's interior temperature was maintained within $1 \mathrm{~K}$ of $12^{\circ} \mathrm{C}$.

The electronics and the thermostat were cooled only via radiation from the Experiment cannister's lid. Thus, too large a heat load on the Experiment cannister would have caused 
a disastrous loss of thermostat control. Covering the cannister lid with appropriate radiator tape and limiting the duration of the Shuttle's Sun-facing orientations prevented this potential problem.

\subsection{Electronics}

\subsubsection{Oscillator drive voltages}

The viscosity was deduced from the ratio of the torque applied to the oscillator to the deflection of the oscillator. Figure 8 is a schematic diagram of the circuit elements that were used to apply the torque and to measure the deflection. The paired $162 \mathrm{k} \Omega$ resistors and $1000 \mathrm{pF}$ capacitors allowed the simultaneous presence at the electrodes of the sub-audio frequency voltage used to drive the oscillator and the $10 \mathrm{kHz}$ voltage used to detect the oscillator's deflection. The $1 \mathrm{M} \Omega$ resistor at the input of the lock-in amplifier grounded the oscillator at low frequencies. A smaller resistor would have decreased the signal-to-noise ratio unacceptably, and a larger resistor would have prevented effective grounding. The interelectrode capacitances $C_{A}, C_{B}, C_{C}$, and $C_{D}$ shown in Figure 8 were approximately 0.3 $\mathrm{pF}$. This was much smaller than the cable capacitance $C_{\text {cable; }}$ however, the $10 \mathrm{kHz}$ signal was still detected with a satisfactory signal-to-noise ratio.

In normal operation, the torque applied to the oscillator was proportional to a timedependent voltage created by summing 400 equal-amplitude sine waves at frequencies evenly spaced from $f_{1}=1 / 32 \mathrm{~Hz}$ to $12.5 \mathrm{~Hz}$,

$$
V_{\mathrm{iII}}(t)=A \sum_{n=1}^{400} \sin \left[2 \pi n f_{1} t+\phi(n)\right] .
$$

This waveform had a period of $32 \mathrm{~s}$. The phases $\phi(n)$ of the 400 components were chosen to minimize the waveform's maximum excursion. A digital-to-analog converter with 16-bit voltage resolution and $512-\mathrm{Hz}$ time resolution generated the waveform. A lowpass filter smoothed the steps in the waveform. Occasionally, low-frequency measurements were made with a similar waveform whose duration was $512 \mathrm{~s}$, and whose lowest frequency was $f_{1}=$ $1 / 512 \mathrm{~Hz}$. 
Because the electrostatic torque was proportional to the square of the drive voltages, we used analog circuits to transform the input voltage $V_{\text {in }}$. A bias voltage $V_{\mathrm{DC}}$ was added to $V_{\text {in }}(t)$, and the square root of the sum,

$$
V_{1}(t)=+V_{\mathrm{DC}}\left[1+\left(V_{\mathrm{in}}(t) / V_{\mathrm{DC}}\right)\right]^{1 / 2},
$$

was obtained. This voltage was applied to one of the diagonally connected electrode pairs. A similar voltage, but of opposite phase,

$$
V_{2}(t)=-V_{\mathrm{DC}}\left[1-\left(V_{\mathrm{in}}(t) / V_{\mathrm{DC}}\right)\right]^{1 / 2},
$$

was applied to the other electrode pair. (The opposite sign of the voltages $V_{1}$ and $V_{2}$ reduced the torque's nonlinearity.) The torque applied to the oscillator was approximately

$$
\frac{C_{A} V_{\mathrm{DC}}^{2} L_{\mathrm{osc}}}{x_{0}}\left[\frac{V_{\mathrm{in}}(t)}{V_{\mathrm{DC}}}+\frac{x(t)}{x_{0}}\right]
$$

where $L_{\text {osc }}$ is the oscillator's length, $x(t)$ is the displacement at the oscillator's tip, and $x_{0} \simeq 4 \mathrm{~mm}$ is the gap between the oscillator and one electrode. The second term of Eq. (2.4) represents a "softening" of the oscillator's spring that lowered the oscillator's resonant frequency $f_{0}$. (See Section 4.3) The square-root circuit and the approximate symmetry of the electrode pairs made the torque on the oscillator very nearly a linear function of $V_{\text {in }}(t)$. The square-root circuit was necessary for linearity because the ratio $V_{\mathrm{in}} / V_{\mathrm{DC}}$ was as large as 0.2 . This ratio could not be decreased significantly by increasing the bias voltage above its actual value, $V_{\mathrm{DC}}=30 \mathrm{~V}$. Such an increase of $V_{\mathrm{DC}}$ would have risked pulling the oscillator against one of the electrode pairs, and it would have added nonlinearity to the oscillator's equation of motion. The lowpass filter following the square-root circuit suppressed noise at $10 \mathrm{kHz}$.

\subsubsection{Oscillator displacement detection}

The oscillator's displacement was detected by the unbalance of a capacitance bridge. See Figure 8 . The bridge was driven by a $3 \mathrm{~V}_{\mathrm{rms}}, 10 \mathrm{kHz}$ oscillator. An inductive voltage divider was adjusted to approximately balance the bridge. The out-of-balance signal was fed to a 
lock-in amplifier which generated a sub-audio frequency voltage $V_{\text {out }}$ that was linear in the difference of the capacitances

$$
\Delta C(t)=\left(C_{A}+C_{D}\right)-\left(C_{B}+C_{C}\right)
$$

This difference was approximately proportional to the oscillator's displacement $x(t)$.

$$
\Delta C(t) \simeq 4 C_{A}\left(\frac{x(t)}{x_{0}}\right) .
$$

The value of $x_{0}$ was a compromise between increasing the sensitivity and increasing the nonlinearity as the gap was decreased.

The viscometry's input and output signals, $V_{\text {in }}$ and $V_{\text {out }}$ were processed in five steps. (1) Anti-alias filters removed frequencies above $128 \mathrm{~Hz}$. (2) The signals were simultaneously digitized at $512 \mathrm{~Hz}$, in synchrony with the digital-to-analog converter which created $V_{\text {in }}(t)$. (3) The resulting time records were Fourier transformed, and all but the 400 lowest frequencies were discarded, thereby digitally filtering the data. (4) The transfer function,

$$
G_{\text {meas }}(f)=\frac{V_{\text {out }}(f)}{V_{\text {in }}(f)}
$$

was computed from the ratio of Fourier transforms of $V_{\mathrm{in}}$ and $V_{\text {out }}$ ) (5) This function was stored as 401 complex numbers. In normal operation, the $32 \mathrm{~s}$ of data collection were followed by $32 \mathrm{~s}$ during which the data were processed, stored, and transmitted to ground. The same $32 \mathrm{~s}$ waveform drove the oscillator during both halves of this $64 \mathrm{~s}$ cycle. The accuracy of the signal processing was verified by constructing a passive lowpass filter with a transfer function that resembled that of the overdamped oscillator. The filter's transfer function measured by the CVX instrument agreed with that measured by a commercial spectrum analyzer (Hewlett Packard 35660A). It also agreed with the transfer function calculated from the values of the filter's components.

The inductive voltage divider shown on Figure 8 was developed by NIST's Electricity Division to fit on a single circuit card. Such a small size was possible because CVX's divider required only nine bits of resolution, much less than the twenty bits typical of commercial programmable dividers. The lock-in amplifier (Ithaco model 410) also fit. on a single card. 


\subsubsection{Electric field effects}

Electric fields drove the oscillator. Thus, they were essential to CVX's operation; however, they had two secondary effects.

The first effect of the electric fields was an increase of the xenon density via electrostriction. Electrostriction was of greatest concern in the immediate vicinity of the oscillator because the oscillator's damping depended approximately upon a weighted integral of $(\eta \rho)^{1 / 2}$ over a volume within a viscous penetration length of the oscillator's surface. Appendix B demonstrates that electrostriction had a negligible influence on CVX's operation because it increased the average density near the oscillator by less than $\Delta \rho / \rho_{c}=0.001$, the uncertainty in the sample's average density.

The second effect of the electric fields was electric-field-driven (dielectrophoretic) convection, which caused parcels of cooler, denser fluid to move toward regions of high electric field. Dielectrophoretic convection is analogous to the buoyancy-driven convection on Earth that transports cooler, denser regions that form near the top of a cell to the bottom of the same cell. In microgravity, such regions formed near the cell's boundary when the cell was cooled, and they formed in the cell's interior when the cell was warmed. Spinodal decomposition also caused their formation when the sample's temperature was brought below $T_{c}$. The characteristic pressure (chemical potential per unit mass) that drove dielectrophoretic convection in the gap between the oscillator and the electrodes was estimated as $P_{E} \approx 0.13$ $\mathrm{mPa}$, four times greater than the hydrostatic pressure caused by microgravity.

\subsubsection{Oscillator amplitude effects}

During the experiment, the oscillator's maximum displacement was $0.03 \mathrm{~mm}$ at the tip of the screen. The resulting product $\dot{\gamma} \tau$ of shear rate $\dot{\gamma}$ and fluctuation-decay time $\tau$ was sufficiently low that CVX did not encounter near-critical shear thinning [23]. The oscillator dissipated approximately $7 \mathrm{pW}$ in the xenon. The resulting rate of density change near the oscillator $\dot{\rho}$ was approximately proportional to the local power per unit volume $\dot{Q}^{\prime}$ and 
inversely proportional to xenon's heat capacity at constant pressure $c_{P}$. The estimate

$$
\dot{\rho}=\frac{(\partial \rho / \partial T)_{P}}{\rho_{\mathrm{c}} c_{P}} \dot{Q}^{\prime}
$$

integrated over the duration of the experiment, was negligible.

Increasing the oscillator's amplitude by a factor of two during ground tests demonstrated that the viscometer's response was independent of amplitude.

\subsubsection{Temperature measurement and control}

Each thermostat shell had an embedded thermistor whose temperature was measured once every eight seconds with a resistance bridge. The shell's temperature was controlled by a proportional-integral-derivative algorithm. The middle shell's temperature was set $0.03 \mathrm{~K}$ below that of the inner shell, and the outer shell was set $0.3 \mathrm{~K}$ below that of the middle shell. A thermistor embedded in the cell's copper wall operated at a power of approximately $0.6 \mu \mathrm{W}$. Its temperature was read by an $\mathrm{AC}$ bridge and lock-in amplifier.

By choosing each bridge's reference resistor to have a value near that of its thermistor at $T_{c}$, the need for an adjustable component, such as a ratio transformer, was eliminated. Temperature was inferred from the bridge's unbalance instead of from the adjustment required to balance the bridge. Far from $T_{c}$, the gain of the lock-in amplifier was decreased to accommodate the bridge's large unbalance. Within $50 \mathrm{mK}$ of $T_{c}$, the rms scatter in the cell's apparent temperature was approximately $10 \mu \mathrm{K}$.

The cell's thermistor was calibrated against the inner shell's thermistor to simplify the cell's temperature control. The inner shell's thermistor calibration consisted of a fit of the Steinhart-Hart equation [24] to the resistances at $0^{\circ} \mathrm{C}, 25^{\circ} \mathrm{C}$, and $50^{\circ} \mathrm{C}$. The manufacturer stated that these three calibration points had an uncertainty of $0.05 \mathrm{~K}$. Thus, the uncertainty of the reduced temperature $t$ was approximately $0.002 t$, and temperature scale nonlinearity contributed negligible error to the derived value of the viscosity exponent. As an independent verification of the calibration's accuracy; the cell's thermistor found $T_{c}=289.721 \mathrm{~K}$. To within $0.02 \mathrm{~K}$, this value is consistent with the value $T_{c}=289.736 \pm 0.006 \mathrm{~K}$ measured 
with a platinum resistance thermometer in the fish tank on the ground and with the value $289.74 \pm 0.02$ quoted elsewhere for xenon [25]. The thermometry's stability was verified by comparing the temperature of the cell $T_{\text {cell }}$ with that of the inner shell $T_{\mathrm{in}}$. The difference $T_{\text {cell }}-T_{\text {in }}$ measured during flight drifted less than $0.1 \mathrm{mK}$ per day, and it differed from the difference measured nine months earlier by only $0.5 \mathrm{mK}$.

\section{Temperature timeline}

The density of a pure fluid near its critical point is extremely sensitive to temperature gradients. For example, at $\rho_{c}$ and $T_{c}+1 \mathrm{mK}$, the isobaric thermal expansivity of xenon is more than a million times larger than that of an ideal gas; thus even a tiny temperature gradient can induce a significant density gradient. In the absence of gravity, this effect limits the fluid's homogeneity. Once formed, the density gradient can be long-lived because microgravity allows very small values of thermal diffusivity $D_{T}$ throughout the sample. At $T_{c}+1 \mathrm{mK}$, the slowest thermal time constant calculated for the CVX sample in the absence of convection was about one week.

CVX's conservative design did not rely on convection to ensure that the density would be sufficiently uniform. Instead, temperature gradients were minimized by careful design of the sample cell, the surrounding thermostat, and the sequence of temperature changes, or "temperature timeline". To keep the sample's density acceptably close to the critical density $\rho_{c}$, the temperature timeline shown in Figure 3 used a two-part strategy. Far from $T_{c}$, the temperature was changed by large, rapid steps, causing large, temporary inhomogeneities in the sample. Each step was followed by a waiting period which exceeded the xenon's longest equilibration time constant and which brought the xenon's density close to $\rho_{c}$. Close to $T_{c}$, although the xenon's temperature was changed without waiting for equilibrium, the density remained sufficiently close to $\rho_{c}$ to obtain meaningful measurements of viscosity.

Candidate timelines were tested with a numerical model of entropy transport within the sample. The model contained two simplifications which allowed efficient testing. First, the xenon sample was modeled as an infinitely long cylinder whose density depended only on the 
radial coordinate. In this approximation, the model was one-dimensional, and heat conduction through the cell's internal parts was ignored. Second, xenon's properties were estimated by an approximation to the cubic model equation of state. (The model is summarized in Reference [2].) In this approximation, terms of order $\theta^{2}$ and higher were dropped to remove the need for iterative calculations. Keeping the state parameter $\theta \ll 1$ made the approximation valid. Figure 9 shows the density deviations calculated for a timeline similar to that used by CVX. Below $T_{c}+100 \mathrm{mK}$, the density deviation in the cell's interior $\left(r / R_{\text {cell }}=0\right)$ is less than $0.13 \%$. Heat conduction through the copper wires that supported the electrodes reduced this to less than $0.06 \%$.

Our confidence in the model's physics came from a recent microgravity experiment [4], in which Wilkinson et al. demonstrated agreement between the measured and calculated values of thermal equilibration time constants in a sample of $\mathrm{SF}_{6}$ near the critical point.

Our confidence in the numerical calculations came from a numerical calculation that could also be described by two analytic calculations. The numerical example calculated the density change $\Delta \rho(t)$ in the sample's interior during and after a $2000 \mathrm{~s}$ ramp of the boundary temperature from $T_{c}+0.8 \mathrm{~K}$ to $T_{c}+1.0 \mathrm{~K}$. During the ramp, $\Delta \rho(t)$ increased until it reached its maximum value at $2000 \mathrm{~s}$. After the ramp, $\Delta \rho(t)$ decayed, becoming exponential after $8000 \mathrm{~s}$. The decay's time constant agreed with the first analytic calculation, which gave $5200 \mathrm{~s}$ for the cylinder's slowest radial mode. The maximum value of $0.0022 \rho_{c}$ agreed with the second analytic calculation. This calculation was the adiabatic upper bound to the density change which occurs in the cell's interior following a sudden change of the cell wall's temperature from $T_{1}$ to $T_{2}$. The bound is

$$
\frac{\Delta \rho}{\rho_{c}} \leq \int_{T_{1}}^{T_{2}}\left(\frac{\partial \rho}{\partial T}\right)_{S} d T \simeq \frac{\left(\rho_{c} / P_{c}\right)}{\left(T_{c} / P_{c}\right)(\partial P / \partial T)_{\rho_{c}}} \int_{T_{1}}^{T_{2}} c_{V} d T
$$

where $c_{V}$ is the constant-volume heat capacity. Eq.(3.1) shows that changing the temperature from $T_{c}+0.1 \mathrm{~K}$ to $T_{c}$ induced changes of density in the sample's interior that were less than $0.2 \%$. Because this constraint is independent of the rate of temperature change, the maximum temperature ramp near $T_{c}$ was limited by other considerations. The ramp rate had to be slow enough that the viscosity would not change significantly during the time 
required to measure it.

The timeline used by CVX included the following features.

1. Each temperature step was followed by a waiting period at constant temperature to observe the sample's approach to equilibrium.

2. The viscometer's calibration data were taken during the waiting period at $T_{c}+1 \mathrm{~K}$.

3. An initial "fast" temperature ramp passed through $T_{c}$ at the rate $-1 \mu \mathrm{K} \cdot \mathrm{s}^{-1}$ and located $T_{c}$ on the cell's thermometer to within $0.1 \mathrm{mK}$.

4. A later series of "slow" temperature ramps, the slowest of which passed through $T_{c}$ at the rate $-0.05 \mu \mathrm{K} \cdot \mathrm{s}^{-1}$ and collected most of the data near $T_{c}$.

\section{Data reduction}

During the mission, most data were downlinked in nearly real time for preliminary analysis, thereby allowing adjustments to the timeline. After the mission, all of the experimental data were retrieved from CVX's hard disk. They comprised approximately $10^{4}$ measurements of the transfer function $G_{\text {meas }}(f)$, plus accompanying temperature measurements. Figure 10 shows typical measurements of magnitude and phase of $G_{\text {meas }}(f)$. After culling and averaging the data, we used a portion of the data from $T_{c}+1 \mathrm{~K}$ to calibrate the viscometer. Viscosity data were then derived from the averaged $G_{\text {meas }}(f)$ by combining the calibration with the model for the oscillator's equation of motion.

After the mission, data farther above $T_{c}$ were collected. For these ground measurements, we calibrated the viscometer at $T_{c}+5 \mathrm{~K}$ instead of at $T_{c}+1 \mathrm{~K}$ due to concerns about internal waves at low frequencies near $T_{c}$ [26]. Appendix $A$ lists viscosity values derived from the microgravity and ground measurements. 


\subsection{Culling and averaging of the data}

The orbital environment influenced CVX in unexpected ways. Figure 11 shows $\left|G_{\text {meas }}(1 \mathrm{~Hz})\right|$ measured during a 24-hour period. The data include oscillations with a 45-minute period and three "spikes" which lasted approximately five minutes each. Such oscillations and spikes occurred throughout the mission, and they affected the magnitude of the transfer function at all frequencies. The oscillations' period was half of the Shuttle's orbital period, which suggests that they were not driven directly either by the Shuttle's exposure to the sun or by the direction of the Earth's magnetic field. In ground tests, exposure of CVX to magnetic field variations comparable to those in orbit did not influence $G_{\text {meas }}(f)$. The minima of the oscillations occurred when the Shuttle was near the equator, and the spikes usually occurred when the Shuttle was near the South Atlantic Anomaly, a region of minimum magnetic field near Argentina. For low Earth orbit, both regions are associated with larger fluxes of the charged particles trapped in the Earth's magnetic field. Thus, the timing of the oscillations and the spikes, and their absence in ground tests of CVX, are consistent with the hypothesis that they were caused by charged particles.

Because the oscillations and the spikes were present only in the magnitude data, they were a time-dependent response of the instrument and not of either the sample or the oscillator. We excluded the spikes from the data, and we suppressed the oscillations by averaging the remaining data in groups of 45 minutes. Each group was thus the average of up to 42 measurements. We did not weight the averaged data by the number of measurements in each group. Instead, we simply excluded from groups containing fewer than 30 measurements. The cell's temperature was averaged in a corresponding manner.

Figure 12 shows that the orbital environment also greatly increased the noise at frequencies below $0.1 \mathrm{~Hz}$. In contrast to the oscillations and the spikes, this very low-frequency noise was present in both the phase and the magnitude, suggesting that either the sample, the oscillator, or both were directly affected. This in-orbit noise prevented our use of the low frequency measurements for deriving the transducer factor $k_{t r} / k_{0}$ (see Section 4.3).

In addition to data adversely affected by the orbital environment, we excluded data taken 
while the sample's density distribution was far from equilibrium. Such data were taken below $T_{c}$ and during and following large steps of temperature.

\subsection{Hydrodynamic similarity and the viscometer's calibration}

The viscometer's calibration exploited a hydrodynamic similarity which applies to the hydrodynamics of an immersed oscillator [27]. This analysis assumed that the fluid was homogeneous and that the oscillator moved as a rigid body. The similarity can be introduced by considering the transfer function of a damped harmonic torsional oscillator. The oscillator's response to a steady sinusoidal force at frequency $f$ is given by

$$
G(f)=\frac{1}{k}\left[1-\left(\frac{f}{f_{0}}\right)^{2}+\frac{i}{Q}\left(\frac{f}{f_{0}}\right)\right]^{-1},
$$

where $k, Q$, and $f_{0}$ are the oscillator's spring constant, quality factor, and undamped resonance frequency respectively, and $G(f)$ is the ratio of the angular displacement of the oscillator to the external torque applied to the oscillator. By giving $Q$ a frequency dependence appropriate for linearized hydrodynamics, this expression can describe the response of an immersed oscillator over a wide range of frequencies. For small amplitude oscillations, the response is

$$
G(f)=\frac{1}{k}\left[1-\left(\frac{f}{f_{0}}\right)^{2}+i\left(\frac{f}{f_{0}}\right)^{2}\left(\frac{\rho}{\rho_{s}}\right) B(R / \delta)\right]^{-1},
$$

where $R$ and $\rho_{s}$ are a characteristic length and density of the oscillator respectively. The function $B(R / \delta)$ characterizes the oscillator's damping in terms of the viscous penetration length, defined by

$$
\delta \equiv \sqrt{\frac{\eta}{\pi \rho f}}
$$

where $\eta$ and $\rho$ are the fluid's viscosity and density. At the nominal frequency of $5 \mathrm{~Hz}$; $\delta \simeq 60 \mu \mathrm{m}$. Thus $\delta$ is larger than the nominal diameter of a screen wire $(2 R=28 \mu \mathrm{m})$, but much less than the distance between wires $(847 \mu \mathrm{m})$.

The viscosity enters $G(f)$ only through the function $B(R / \delta)$. Most oscillating-body viscometers are sensitive to viscosity changes only in a narrow range of frequencies near $f_{0}$. 
and $B(R / \delta)$ must be calculated from the hydrodynamic theory for an idealized geometry. In contrast, we determined $B(R / \delta)$ by measuring $G(f)$ when the xenon was in a reference state of known $\eta$ and $\rho$ far from $T_{c}$ and then inverting Eq.(4.6) below. This procedure calibrated the viscometer for changes in the viscosity with respect to the reference viscosity. We emphasize that this calibration did not require knowledge of the geometry of either the oscillator or the surrounding electrodes, and it was indifferent to the choices of the parameters $R$ and $\rho_{s}$. The accuracy of this technique was demonstrated in Reference [27]. There, the viscosity of carbon dioxide was measured using a viscometer much like the CVX viscometer, and the results agreed with accurate published data acquired by conventional means.

The CVX viscometer's calibration was derived during flight from an average of four 45-minute measurements of $G(f)$ near the temperature $T_{c}+1 \mathrm{~K}$. At this temperature, we equated the viscosity with that determined by the high- $Q$ torsion oscillator [10], which had an uncertainty of $0.8 \%$. After applying the corrections described below, useful values of $B(R / \delta)$ were derived from the transfer function at frequencies from 0.3 to $12.5 \mathrm{~Hz}$, corresponding to a range of $\delta$ much broader than that encountered upon approaching $T_{c}$ at fixed frequency. We represented the magnitude and phase of $B(R / \delta)$ by polynomial functions of $\ln (R / \delta)$ in the range $0.05<R / \delta<0.4$.

Figure 13 shows the measured values of $B(R / \delta)$ and an approximation to $B(R / \delta)$ derived from an analytical model of the oscillator. This model is Stokes' solution for the viscous force exerted on a transversely oscillating cylinder. We used the modern formulation [28],

$$
B_{c y l}(R / \delta)=-i\left[1+\frac{4 K_{1}(z)}{z K_{0}(z)}\right]
$$

where $K_{n}(z)$ is the modified Bessel function of order $n$, and $z \equiv(1+i)(R / \delta)$.

\subsection{Corrections to the ideal transfer function}

Figure 10 shows an example of the measured transfer function $G_{\text {tneas }}(f)$. Several effects caused $G_{\text {meas }}(f)$ to differ from the response $G(f)$ given by Eq.(4.2). The largest effect was due to the driving and detection electronics shown in Figure 8, which acted like a lowpass 
filter with a transfer function $G_{\text {elec }}$. The transfer function of the electronics $G_{\text {elec }}$ was obtained by disconnecting the driving and detection stages from the cell and measuring their transfer functions independently.

The oscillator's torsion spring was not ideal because slow relaxation processes within the nickel itself caused internal friction and creep. At the low amplitudes used in our measurements, these phenomena could be described as linear anelastic effects [21]. We corrected for anelasticity by generalizing the spring constant $k$ to a complex function of frequency

$$
k=k_{0}[1+\psi(f)+i \phi(f)]
$$

where $k_{0}$ is the real part of the spring constant at the resonance frequency. The real functions $\psi(f)$ and $\phi(f)$, were measured in vacuum from 0.001 to $10 \mathrm{~Hz}$ at several temperatures [21].

The oscillator's resonance frequency $f_{0}$ was corrected for its dependence on temperature and for the electrostatic spring softening induced by the DC voltage $V_{\mathrm{DC}}$ applied to the cell's electrodes. Both effects could be measured in vacuum only. Due to xenon's dielectric constant, we expected a $0.4 \%$ decrease of $f_{0}$ upon filling the cell, and this was included in the correction to $f_{0}$. (The function $B(R / \delta)$ accounted for the xenon's effect on the oscillator's hydrodynamic mass.)

We did not correct $G_{\text {rneas }}(f)$ to account for a second mode of the oscillator at higher frequency. A mode near $54 \mathrm{~Hz}$, near the calculated bending frequency, was seen in vacuum measurements of a similar oscillator. However, no such mode was seen below $100 \mathrm{~Hz}$ with the CVX oscillator. Perhaps the bending mode was shifted to higher frequencies by stiffening caused by a slight crease of the nickel screen.

With these corrections, the transfer function becomes

$$
G_{\text {tneas }}(f)=G_{\text {elec }}(f)\left(\frac{k_{t r}}{k_{0}}\right)\left[1+\psi(f)+i \phi(f)-\left(\frac{f}{f_{0}}\right)^{2}+i\left(\frac{f}{f_{0}}\right)^{2}\left(\frac{\rho}{\rho_{s}}\right) B(R / \delta)\right]^{-1},
$$

where $k_{t r}$ is a real constant given by the product

$$
k_{t r}=\left(\frac{\text { oscillator torque }}{\text { input voltage }}\right)\left(\frac{\text { output voltage }}{\text { oscillator displacement }}\right)\left(\frac{1}{G_{\text {eloc }}}\right) .
$$


The product of transducer factors $k_{t r}$ related the measured voltages $V_{\text {in }}$ and $V_{\text {out }}$ to the oscillator's torque and displacement. We assumed that $k_{t r}$ was independent of frequency.

Determination of the ratio $k_{t r} / k_{0}$ requires accurate knowledge neither of $k_{0}$ nor of the transducer factors. For example, in ground tests we obtained $k_{t r} / k_{0}$ from measurements of $G_{\text {meas }}(f)$ made near $0.01 \mathrm{~Hz}$, where viscous damping was insignificant. Derivation of the value

$$
\frac{k_{t r}}{k_{0}} \equiv \frac{\left|G_{\text {meas }}(0.01 \mathrm{~Hz})\right|}{1-\psi(0.01 \mathrm{~Hz})}
$$

required knowledge only of the real part of the anelastic correction. In orbit, we were unable to measure $k_{t r} / k_{0}$ in a similar fashion because the signal-to-noise ratio of $G_{\text {meas }}(0.01 \mathrm{~Hz})$ decreased by a factor of 10 or more. Instead, we set $k_{t r} / k_{0}$ to the value that made $\operatorname{Re}(\eta)$ independent of frequency for $f>1 \mathrm{~Hz}$ in the temperature range $3 \times 10^{-5}<t<5 \times 10^{-2}$ where viscoelastic effects were absent. The in-orbit and ground values of $k_{t r} / k_{0}$ agreed within $0.3 \%$.

The parameter $k_{t r} / k_{0}$ had linear dependences on the Experimental cannister's temperature, the cell's temperature, and time. The effects of these dependences can be seen in Figure 14, which is a plot of $\left|G_{\text {meas }}(0.5 \mathrm{~Hz})\right|$. At $0.5 \mathrm{~Hz},\left|G_{\text {meas }}\right|$ is nearly independent of the sample's viscosity; thus, only unintentional dependences are present. The dependence on the Experimental cannister's temperature probably originated in the electronics; its coefficient was determined in ground tests. The dependence on the cell's temperature was assumed to result from the temperature dependence of the elastic constant of the torsion fiber and the thermal expansion of the capacitors. The torsion constant's temperature dependence was inferred from measurements of $d f_{0} / d T$, and changes in the capacitance were delermined by fitting $\left|G_{\text {meas }}(0.5 \mathrm{~Hz})\right|$ to a linear function of the cell's temperature. The coefficient of the linear time dependence was determined by fitting to $\left|G_{\text {nneas }}(0.5 \mathrm{~Hz})\right|$. The origin of this time dependence was unknown. However, a similar time dependence occurred in $\left|G_{\text {meas }}(0 \mathrm{~Hz})\right|$ : which was linear in the oscillator's average displacement; both time dependences may have resulted from creep in the oscillator's equilibrium position. 
( $\left.T-T_{\text {cal }}\right)$ raised to a small power such as $z_{\eta}$, all of our results would have been unchanged.

As an additional check on the data reduction, we systematically varied various data reduction parameters from their nominal values. We changed the density by $1 \%$ from $\rho_{c}$. We changed the calibration viscosity by $3 \%$. We changed the oscillator's vacuum resonance frequency by $3 \%$ from $f_{0}$. We perturbed the transfer function $G_{\text {elec }}$ used to describe the electronics. None of these variations caused significant changes in the data's consistency.

\section{Results}

\subsection{Sample homogeneity}

\subsubsection{History independence}

Density inhomogeneities that were induced by temperature changes caused no problems. Figure 15 supports this claim by showing the oscillator's phase measured at $2 \mathrm{~Hz}$ during a tenday period. On Figure 15, measurements made at $300 \mathrm{mK}, 100 \mathrm{mK}, 30 \mathrm{mK}$, and $3 \mathrm{mK}$ above $T_{\mathrm{c}}$ are highlighted by boxes. At each temperature, measurements were repeated as much as seven days later and with very different temperature histories. The phase measurements agreed to within $1 \mathrm{mrad}$. This corresponds to an agreement in density of $0.1 \%$.

Figure 4 shows that the sample's density could be kept near $\rho_{c}$ even within $0.1 \mathrm{mK}$ of $T_{c}$. Data collected during three ramps through $T_{c}$ are shown. The slowest ramp, at $-0.05 \mu \mathrm{K} \cdot \mathrm{s}^{-1}$; collected most of the data near $T_{c}$. The medium ramp, at $-1 \mu \mathrm{K} \cdot \mathrm{s}^{-1}$, was intended only to locate $T_{c}$ to within $1 \mathrm{mK}$. Nevertheless, it gave results above $T_{c}$ that were indistinguishable from those of the slowest ramp. Furthermore, after culling data points as described earlier, the data at higher temperatures also were independent of the sample's temperature history.

On Figure 4, data from the fastest ramp at $-33 \mu \mathrm{K} \cdot \mathrm{s}^{-1}$ are displaced above and to the left of the equilibrium data. Because this ramp started at $T_{c}+0.2 \mathrm{~K}$, Eq.(3.1) permits the sample's interior density to decrease by up to $0.3 \%$. This decrease is consistent with the data's vertical displacement. (Also, the thermometer's lag of several seconds caused a horizontal displacement of $0.1 \mathrm{mK}$.) Note also the ramp-rate dependence of the phase at 2 
$\mathrm{Hz}$ below $T_{c}$. This time dependence is consistent with phase separation.

\subsubsection{Equilibration}

When the cell's temperature was held constant, the viscosity rapidly approached a steady value, typically within one hour. (See Figure 15.) Even after accounting for heat conduction through the cell's metal electrodes, this was approach was faster than expected from the xenon's thermal diffusivity. At $300 \mathrm{mK}, 100 \mathrm{mK}, 30 \mathrm{mK}$, and $3 \mathrm{mK}$ above $T_{c}$, the sample's calculated time constants were $1 \mathrm{~h}, 2 \mathrm{~h}, 6 \mathrm{~h}$, and $30 \mathrm{~h}$, respectively.

Three phenomena contributed to the rapid approaches to equilibrium. First, convection driven by gravity and by electric fields slowly stirred the xenon. The stirring was effective because the open geometry around the oscillator reduced viscous damping of the convective flow. For example, if a density deviation $\Delta \rho=0.01 \rho_{c}$ had occurred in a region of characteristic size $L=4 \mathrm{~mm}$, an acceleration $10^{-6}$ of Earth's gravity would have turned over that portion of the sample in a time of order $\eta /(g L \Delta \rho) \approx 3$ minutes. Second, the oscillator was thermally isolated from the cell wall, and it had negligible heat capacity. Thus, the deviation of the density caused by a temperature step above $T_{c}$ was equal to that of the cell's interior (Eq.(3.1)) and not of the much larger deviation that occurred near the cell's boundaries. For example, the initial density deviation in the cell's interior following the steps from $T_{c}+300$ $\mathrm{mK}$ to $T_{c}+100 \mathrm{mK}$ was only $0.3 \%$. Third, the oscillator was insensitive to deviations of the density from $\rho_{c}$ if the deviations averaged to zero over the oscillator's surface. This insensitivity occurred because the oscillator's response was approximately proportional to the surface-averaged value of $\sqrt{\eta p}$ and, above $T_{c}+1 \mathrm{mK}$ and for small deviations from $\rho_{c}$, the viscosity is proportional to the density.

To demonstrate the viscometer's sensitivity to density inhomogeneities, we deliberately created an inhomogeneous sample by cooling the cell to $T_{c}-9 \mathrm{mK}$ and then warming it to $T_{c}+0.5 \mathrm{mK}$. We then observed the apparent viscosity for four hours. This set of observations is labeled "B" and enclosed by an oval on Figure 15. It can be compared to the set labeled "A" that was cooled to $T_{c}-0.5 \mathrm{mK}$ and then warmed to $T_{c}+3 \mathrm{mK}$. Because xenon's thermal 
diffusivity at $T_{c}+0.5 \mathrm{mK}$ is three times smaller than at $T_{c}+3 \mathrm{mK}$, one might expect the approach to equilibrium of set $B$ to be three times slower than the approach of set A. Instead, the approach of set $B$ was slower by much more than a factor of three; the apparent viscosity differed from the equilibrium viscosity by $5 \%$ at the end of the four-hour observation.

One contribution to set B's slower equilibration was the limited thermal conductance of the copper wire that connected each electrode to the cell wall. Below approximately $T_{c}+1 \mathrm{mK}$, the conductance of the copper wire was exceeded by that of the xenon between the electrode and the cell wall, and the length $L_{\text {thermal }}$ that governed the sample's thermal diffusion was the cell's $19 \mathrm{~mm}$ diameter. In contrast, $L_{\text {thermal }}$ for set A was the $8 \mathrm{~mm}$ gap between the electrodes.

Another possible contribution to set B's slower equilibration was the size of its inhomogeneities. They were formed at $T_{c}-9 \mathrm{mK}$, where surface-tension-driven fows ensured that the liquid and vapor regions quickly grew as large as the sample's dimensions. In contrast, set A's inhomogeneities were formed between $T_{c}$ and $T_{c}-0.5 \mathrm{mK}$ during a two-hour period; this is sufficiently brief that the liquid and vapor regions may not have had enough time to grow as large as the $8 \mathrm{~mm}$ interelectrode gap [7]. In that case, $L_{\text {thermal }}$ was limited to the size of set A's inhomogeneities, thereby speeding the decay of the inhomogeneities by thermal diffusion.

\subsection{Fits to the data}

\subsubsection{Model of the data}

Our model of the frequency-dependent data was Eq.(1.2). The frequency scaling function $S(z)$, including corrections to two typographic errors in Ref. [8], is given in Appendix C. We introduced the parameter $A$ into the argument of the scaling function to obtain agreement with our data. Setting $A \equiv 1$ caused large systematic deviations from the fits.

In Eq.(1.2), the background viscosity was described by the sum of analytic functions of the density and the temperature:

$$
\eta_{0}(T, \rho)=\eta_{00}(T)+\eta_{01}(\rho) .
$$


These functions were determined from the viscosity measured by others far from the critical point. (See Appendix C of Reference [10].) For consistency with the measurements of Reference [10], we set $\eta_{0}\left(T_{c}, \rho_{c}\right)=(51.3 \pm 0.4) \mu \mathrm{Pa} \cdot \mathrm{s}$. The present results are not sensitive to this description of $\eta_{0}$. We used the value $\rho_{c}=1116 \mathrm{~kg} \cdot \mathrm{m}^{-3}$ determined from Närger and Balzarini's Sample No. 1 [29]. Both Sample No.1 and CVX used high purity xenon from Matheson. We estimated the uncertainty of $\rho_{c}$ as the difference of $1.3 \mathrm{~kg} \cdot \mathrm{m}^{-3}$ between the values for Samples No. 1 and 2.

Ideally the exponent characterizing the divergence of any thermophysical property would be determined from data so close to $T_{c}$ that the critical anomaly is much larger than the noncritical background. The small value of the exponent $z_{\eta}$ makes this impossible for viscosity, and the most accurate determination of $z_{\eta}$ from the data requires knowledge of the background viscosity $\eta_{0}$ and of the crossover function $H(\xi)$. Recently, Luettmer-Strathmann, Sengers, and Olchowy $[30,31]$ published equations designed to describe the crossover behavior of both viscosity and thermal conductivity with a single fluid-dependent wave-number cutoff $q_{D}$. We used the older crossover function by Bhattacharjee et al. [9] for viscosity because it is simpler and has the same asymptotic behavior as the newer function. The older crossover function makes approximations not used in the newer function. The chief approximation is neglect of the divergence in the constant-volume heat capacity $c_{V}$ [32]. Because we fitted to data only at reduced temperatures less than $10^{-3}$, where the heat capacity ratio $c_{V} / c_{P}<0.002$, this approximation introduced negligible errors.

The following subsections describe the results for $T_{c}, z_{\eta}, A, q_{c}$, and $q_{D}$ obtained by fitting the model to the viscosity data. The fit included the values of $\operatorname{Re}(\eta)$ and $\operatorname{Im}(\eta)$ measured at $2 \mathrm{~Hz}, 3 \mathrm{~Hz}, 5 \mathrm{~Hz}, 8 \mathrm{~Hz}$, and $12 \mathrm{~Hz}$ within the range $10^{-6}<t<10^{-4}$. Expanding the range of fitted data to $10^{-6}<t<10^{-3}$ caused negligible changes in the fitted parameters, while slightly increasing the fit's systematic deviations: The value of reduced chi-squared increased from 0.97 to 1.05 . 


\subsubsection{Viscosity cusps near $T_{c}$}

The fit ignored the data within $0.3 \mathrm{mK}$ of $T_{c}$ and yielded $T_{c}=(16.571237 \pm 0.000008){ }^{\circ} \mathrm{C}$. The standard uncertainties quoted here and below represent a coverage factor of one $(68 \%$ confidence interval), and they allow for the correlations among the parameters. (We recall that our thermometer's uncertainty with respect to ITS90 was $50 \mathrm{mK}$.) The phase and magnitude data exhibited cusps close to the fitted value of $T_{c}$. The minimum in the phase at $2 \mathrm{~Hz}$ shown in Figure 4 is an example of such a cusp. As illustrated in Figure 16, the phase at frequencies higher than $2 \mathrm{~Hz}$ exhibited a sharp local maximum. Table 1 lists the temperatures of three example cusps obtained by separate fits to data in a $2 \mathrm{mK}$ span that included $T_{c}$.

\begin{tabular}{|l|l|}
\hline \multicolumn{2}{|c|}{ Table 1: Cusps near $T_{c}$} \\
\hline Cusp & $\left(T_{\text {cusp }}-T_{c}\right) / \mu \mathrm{K}$ \\
\hline phase at $2 \mathrm{~Hz}$ & +56 \\
\hline magnitude at $5 \mathrm{~Hz}$ & -1 \\
\hline phase at $5 \mathrm{~Hz} 5 \mathrm{~Hz}$ & -17 \\
\hline
\end{tabular}

Each value of $T_{\text {cusp }}$ was determined to within $10 \mu \mathrm{K}$. The values of $T_{\text {cusp }}$ are thus mutually inconsistent even though they all differ from $T_{c}$ by less than $60 \mu \mathrm{K}$. We allowed $T_{c}$ to be a fitted parameter because $T_{c} \neq T_{\text {cusp }}$. Viscoelasticity and time-dependent phase separation determine $T_{\text {cusp }}$.

\subsubsection{Viscosity exponent $z_{\eta}$}

The viscosity exponent was obtained by dividing the fitted value, $\nu z_{\eta}=0.04349 \pm 0.00035$, by the correlation length exponent, $\nu=0.630,[33,34]$ to obtain $z_{\eta}=0.0690 \pm 0.0006$. This value for $z_{\eta}$ is $7 \%$ larger than the value obtained from the high- $Q$ torsion oscillator [10], even though the two data sets agree at $t>3 \times 10^{-4}$, where gravity had negligible influence on the high- $Q$ data, and even though both data sets were analyzed with the same crossover function. The high- $Q$ data led to a lower value of $z_{\eta}$ because they were obtained at larger reduced 
temperatures, where the inaccuracy of the crossover function used here and in Ref. [10] is more important. Consistent with this attribution, fits to the present data at larger reduced temperatures yielded smaller values of $z_{\eta}$. These fits also showed systematic deviations that would not have been detected in the high- $Q$ data because the latter had more noise.

Two recent papers by Hao, Ferrell, and Bhattacharjee have refined the theoretical estimate of $z_{\eta}$. The first paper [35] is a mode coupling calculation of $z_{\eta}$ in a two-term epsilon expansion. In contrast to earlier work by Siggia et al. [36], Hao et al. found that vertex corrections were significant, and they argued that a two-loop perturbation expansion in three dimensions would give a more accurate result. The second paper [15] gives the more accurate calculation, which leads to $z_{\eta}=0.067 \pm 0.002$, where the uncertainty is the authors' "rough estimate of the inaccuracy introduced by various approximations". Thus the experimental and theoretical values of $z_{\eta}$ agree within their combined uncertainties.

\subsubsection{Viscoelastic time scale $A$}

The value of $A$ was obtained by replacing the fluctuation-decay time $\tau_{0}$ with the free parameter $\tau_{0}^{\prime}$. This procedure yielded $\tau_{0}^{\prime}=(2.31 \pm 0.06) \mathrm{ps}$, thereby determining the frequency scale $A z$ with a relative uncertainty of 0.03 . However, the relative uncertainty of the ratio $A \equiv \tau_{0}^{\prime} / \tau_{0}=2.0 \pm 0.3$ is 0.15 because of the larger uncertainty of the fluctuationdecay time amplitude $\tau_{0}$, given by Eq.(1.1). For xenon, $\tau_{0}=(1.15 \pm 0.17) \mathrm{ps}$, and the uncertainty of $\tau_{0}$ is dominated by the uncertainty of xenon's correlation length amplitude, $\xi_{0}=(0.184 \pm 0.009) \mathrm{nm}$, which we estimated as the difference between the two measurements reported by Güttinger and Cannell [37].

Bhattacharjee and Ferrell's theory of near-critical viscoelasticity $[38,8]$ was stimulated by Bruschi et al.'s high-frequency measurements of viscosity near the critical point of $\mathrm{CO}_{2}$ [11]. These measurements, and later measurements by Bruschi in $\mathrm{CO}_{2}$ [12] and by Izumi et al. in xenon [13], showed only qualitative agreement with theory. Berg and Moldover's single-frequency measurements of two binary liquids required viscoelasticity in the analysis. (See Figure 9 in [14].) However, the magnitude of the observable viscoelastic effect was only 
$0.5 \%$, in hindsight we see that their data are consistent with $A>1$.

In contrast to these previous measurements, the present data provide a quantitative test of the predicted functional forms. As shown by Figure 2, the functional form $S(A z)$ describes the data for both $\operatorname{Re}(\eta)$ and $\operatorname{Im}(\eta)$ over a wide range of scaled frequency $(0.0004<A z<33)$, provided that the viscoelastic time scale is adjusted by the factor $A=2.0 \pm 0.3$. In particular, the description is consistent with the prediction that $\operatorname{Im}(\eta) / \operatorname{Re}(\eta)$ approaches 0.035 for large values of $z$.

\subsubsection{Wavevectors $q_{C}$ and $q_{D}$}

Fitting to the logarithms $\ln \left(q_{C} \xi_{0}\right)$ and $\ln \left(q_{D} \xi_{0}\right)$ instead of $q_{C} \xi_{0}$ and $q_{D} \xi_{0}$ made the fitting routine more robust. The values obtained were $q_{C} \xi_{0}=0.051 \pm 0.007$ and $q_{D} \xi_{0}=0.16 \pm 0.05$. The fitted value of $q_{C} \xi_{0}$ agrees with the independent value $0.059 \pm 0.004$ determined from published data that do not include the near-critical viscosity. See Appendix D.

\section{Acknowledgements}

We thank the CVX development team led by A.M. Peddie and J.L. Myers at NYMA Inc., the TAS-01 Mission team led by N.F. Barthelme, and the STS- 85 crew. We thank R.A. Ferrell, R.W. Gammon, and R.A. Wilkinson for stimulating and critical comments. The CVX project was managed by R.W. Lauver and I. Bibyk through the NASA Lewis Research Center and was funded by the NASA Microgravity Science and Applications Division.

\section{A. Appendix: Tablulated viscosity data}

Table $A 1$ and $A 2$ give values of $\operatorname{Re}(\eta)$ outside the viscoelastic region. Only data taken at 2 $\mathrm{Hz}$ are listed. The data at higher frequencies were consistent with those at $2 \mathrm{~Hz}$; however, they had smaller signal-to-noise ratios. Some of the values represent a single 45-minute data point, and their uncertainty $\sigma_{1}$ was estimated as the standard deviation of all of the 
45-minute points from a fit to the data in the range $10^{-6}<t<10^{-3}$. The other values are averages of $N$ 45-minute points, and their uncertainty was estimated as $\sigma_{1} / \sqrt{N}$.

Table A3 gives values of $\operatorname{Re}(\eta)$ and $\operatorname{Im}(\eta) / \operatorname{Re}(\eta)$ inside the viscoelastic region. Each temperature represents a single 45 -minute data point. 
Table A1

MICROGRAVITY

\begin{tabular}{cc}
\hline $10^{5} t$ & $\operatorname{Re}[\eta(2 \mathrm{~Hz})]$ \\
& $(\mu \mathrm{Pa} \cdot \mathrm{s})$ \\
\hline 1029.0 & $52.241 \pm 0.017$ \\
344.3 & $53.009 \pm 0.018$ \\
104.1 & $54.509 \pm 0.010$ \\
100.0 & $54.585 \pm 0.013$ \\
96.35 & $54.576 \pm 0.022$ \\
65.51 & $55.213 \pm 0.018$ \\
59.60 & $55.275 \pm 0.032$ \\
43.36 & $55.923 \pm 0.023$ \\
35.89 & $56.305 \pm 0.010$ \\
35.48 & $56.339 \pm 0.010$ \\
34.76 & $56.349 \pm 0.013$ \\
29.69 & $56.709 \pm 0.012$ \\
21.18 & $57.359 \pm 0.024$ \\
20.76 & $57.389 \pm 0.015$ \\
17.07 & $57.824 \pm 0.012$ \\
14.93 & $58.080 \pm 0.017$ \\
11.73 & $58.658 \pm 0.017$ \\
11.03 & $58.784 \pm 0.024$ \\
8.645 & $59.291 \pm 0.024$ \\
8.620 & $59.351 \pm 0.011$ \\
6.808 & $59.876 \pm 0.025$ \\
5.886 & $60.246 \pm 0.014$ \\
4.978 & $60.610 \pm 0.025$ \\
4.381 & $60.937 \pm 0.018$ \\
3.142 & $61.765 \pm 0.025$ \\
2.960 & $61.930 \pm 0.011$ \\
2.211 & $62.713 \pm 0.018$ \\
\hline & \\
&
\end{tabular}

Table 42 GROUND

\begin{tabular}{cc}
\multicolumn{2}{c}{ GROUND } \\
\hline $10^{5} t$ & $\begin{array}{c}\operatorname{Re}[\eta(2 \mathrm{~Hz})] \\
(\mu \mathrm{Pa} \cdot \mathrm{s})\end{array}$ \\
\hline 5527. & $52.623 \pm 0.015$ \\
3976. & $52.316 \pm 0.012$ \\
2420. & $52.073 \pm 0.017$ \\
1730. & $52.047 \pm 0.021$ \\
1729. & $52.048 \pm 0.010$ \\
1729. & $52.048 \pm 0.010$ \\
1729. & $52.047 \pm 0.010$ \\
1729. & $52.053 \pm 0.017$ \\
1039. & $52.178 \pm 0.017$ \\
1034. & $52.162 \pm 0.017$ \\
354.2 & $52.928 \pm 0.031$ \\
338.0 & $52.974 \pm 0.015$ \\
220.9 & $53.446 \pm 0.031$ \\
140.9 & $54.011 \pm 0.022$ \\
105.6 & $54.375 \pm 0.011$ \\
105.4 & $54.360 \pm 0.010$ \\
\hline
\end{tabular}




\section{Table A 3}

\begin{tabular}{|r|rrrrr|rrrrrrr|}
\hline $10^{6} t$ & \multicolumn{7}{|c|}{$\mathrm{Re}(\eta) /(\mathrm{mPa} \cdot \mathrm{s})$} & \multicolumn{5}{c|}{$-10^{4} \mathrm{Im}(\eta) / \mathrm{Re}(\eta)$} \\
\hline & $2 \mathrm{~Hz}$ & $3 \mathrm{~Hz}$ & $5 \mathrm{~Hz}$ & $\mathbf{8 H z}$ & $12 \mathrm{~Hz}$ & $2 \mathrm{~Hz}$ & $3 \mathrm{~Hz}$ & $5 \mathrm{~Hz}$ & $8 \mathrm{~Hz}$ & $12 \mathrm{~Hz}$ \\
& \pm 0.04 & \pm 0.04 & \pm 0.05 & \pm 0.07 & \pm 0.11 & \pm 8 & \pm 6 & \pm 4 & \pm 8 & \pm 15 \\
\hline 19.91 & 63.00 & 63.00 & 62.99 & 63.04 & 62.92 & 5 & 9 & 7 & 17 & 11 \\
18.91 & 63.14 & 63.13 & 63.12 & 63.09 & 62.94 & 6 & 8 & -1 & 9 & 4 \\
16.63 & 63.46 & 63.47 & 63.41 & 63.51 & 63.47 & 7 & 2 & 8 & 25 & 7 \\
15.70 & 63.62 & 63.64 & 63.63 & 63.60 & 63.55 & 9 & 7 & 11 & 12 & 28 \\
13.80 & 63.96 & 63.97 & 63.99 & 63.91 & 63.95 & 5 & 7 & 15 & 17 & -13 \\
12.72 & 64.18 & 64.18 & 64.17 & 64.23 & 63.93 & -8 & 9 & 12 & 31 & 15 \\
11.92 & 64.33 & 64.36 & 64.36 & 64.37 & 64.19 & 5 & 13 & 18 & 12 & 10 \\
11.03 & 64.53 & 64.53 & 64.50 & 64.50 & 64.36 & 2 & 12 & 11 & 31 & 23 \\
10.27 & 64.71 & 64.72 & 64.70 & 64.66 & 64.66 & 9 & 14 & 18 & 42 & 56 \\
9.76 & 64.83 & 64.84 & 64.84 & 64.85 & 64.62 & 5 & 15 & 20 & 29 & 51 \\
9.27 & 64.96 & 64.99 & 64.98 & 64.99 & 64.79 & 5 & 12 & 24 & 39 & 58 \\
8.84 & 65.13 & 65.11 & 65.06 & 65.06 & 64.81 & 5 & 15 & 16 & 50 & 3 \\
8.38 & 65.27 & 65.26 & 65.26 & 65.16 & 65.27 & 2 & 15 & 22 & 47 & 24 \\
7.87 & 65.43 & 65.42 & 65.40 & 65.40 & 65.35 & 9 & 17 & 32 & 43 & 17 \\
7.41 & 65.58 & 65.57 & 65.53 & 65.48 & 65.24 & 3 & 19 & 26 & 53 & 72 \\
6.97 & 65.77 & 65.79 & 65.70 & 65.71 & 65.57 & 18 & 21 & 20 & 40 & 45 \\
6.21 & 66.09 & 66.08 & 66.05 & 65.95 & 65.83 & 17 & 28 & 40 & 64 & 63 \\
5.34 & 66.47 & 66.49 & 66.38 & 66.41 & 66.11 & 22 & 32 & 43 & 62 & 62 \\
4.88 & 66.77 & 66.74 & 66.68 & 66.63 & 66.56 & 39 & 45 & 57 & 82 & 76 \\
4.40 & 67.00 & 67.01 & 66.93 & 66.90 & 66.43 & 28 & 44 & 58 & 92 & 115 \\
3.95 & 67.31 & 67.30 & 67.28 & 67.12 & 66.81 & 38 & 56 & 69 & 94 & 103 \\
3.49 & 67.64 & 67.60 & 67.46 & 67.25 & 67.11 & 48 & 63 & 71 & 107 & 118 \\
3.02 & 68.00 & 67.95 & 67.81 & 67.59 & 67.45 & 55 & 79 & 84 & 113 & 111 \\
2.60 & 68.37 & 68.25 & 68.17 & 67.93 & 67.51 & 77 & 98 & 102 & 145 & 123 \\
2.19 & 68.82 & 68.74 & 68.53 & 68.19 & 67.58 & 90 & 110 & 131 & 157 & 175 \\
1.69 & 69.38 & 69.24 & 68.92 & 68.56 & 68.22 & 111 & 131 & 155 & 178 & 222 \\
1.28 & 69.94 & 69.71 & 69.39 & 68.85 & 68.60 & 143 & 169 & 187 & 207 & 198 \\
0.79 & 70.65 & 70.33 & 69.81 & 69.36 & 68.68 & 179 & 213 & 237 & 265 & 253 \\
0.31 & 71.43 & 70.98 & 70.39 & 69.71 & 69.05 & 268 & 290 & 300 & 319 & 306 \\
\hline
\end{tabular}




\section{B. Appendix: Electrostriction}

The relative increase in the density $\Delta \rho / \rho_{c}$ caused by a time-independent, electric field $E$ is

$$
\frac{\Delta \rho}{\rho_{\mathrm{c}}}=\left(\frac{P_{E}}{P_{\mathrm{c}}}\right) \chi_{T}^{*},
$$

where $P_{E}=\left(\epsilon_{\mathrm{Xe}} / \epsilon_{0}-1\right) \epsilon_{0} E^{2} / 2$ is the induced change in chemical potential per unit mass, $P_{c}$ is xenon's critical pressure, and $\epsilon_{0}$ and $\epsilon_{\mathrm{Xe}}$ are the dielectric constants of vacuum and xenon. Electrostriction is conspicuous near $T_{c}$ because of the large density changes resulting from the large value of $\chi_{T}^{*}$, the reduced susceptibility at constant temperature.

As a first step toward calculating $E$, we ignored the oscillator's presence and modeled the electric field between the viscometer's driving electrodes as that in a parallel plate capacitor. See Figure 17. If the voltages on the two electrodes are $V_{1}$ and $V_{2}$, and the distances from the oscillator to the two electrodes are $x_{1}$ and $x_{2}$, this electric field is

$$
E_{0}=\frac{\left(V_{1}-V_{2}\right)}{\left(x_{1}+x_{2}\right)} .
$$

Electrostriction due to $E_{0}$ was insignificant.

Next we modeled the field concentrated near one of the thin wires comprised by the oscillator. We adapted Spangenberg's [39] model for the electric potential in a vacuum tube, consisting of two electrodes and a grid modeled as planar array of parallel line charges. The radial field $E_{r}$ near one of the wires, modeled as a cylinder, is

$$
E_{r}(r, \theta)=\left(E_{1}+E_{2}\right)\left(\frac{a}{4 \pi r}\right)+E_{0}\left[1+\left(\frac{R}{r}\right)^{2}\right] \cos (\theta) .
$$

Here $r$ and $\theta$ are cylindrical coordinates centered on the wire, $R$ is the radius of the wire, and $a \gg R$ is the spacing between wires. The first term in Eq.(B.3) originates in the wire's induced line charge, and it disappears when the fields $E_{1} \equiv V_{1} / x_{1}$ and $E_{2} \equiv V_{2} / x_{2}$ are equal and opposite. It is a factor of two smaller than in Spangenberg's model because the charge induced on the CVX oscillator was spread over transverse as well as longitudinal wires. The second term originates in the wire's induced line dipole; it was added to Spangenberg's model to create a zero potential surface at $r=R$. 
We applied Eq.(B.3) to CVX using the values $a=0.85 \mathrm{~mm}, R=0.009 \mathrm{~mm}$, and $V_{1}=-V_{2}=V_{\mathrm{DC}}=30 \mathrm{~V}$. We allowed for asymmetry in the viscometer's construction by estimating the unequal distances $x_{1}=3 \mathrm{~mm}$ and $x_{2}=5 \mathrm{~mm}$. At the wire's surface, the field was concentrated by the factor $E_{r} / E_{0}=6$. At $T_{c}+0.3 \mathrm{mK}$, the associated density increase was only $0.05 \%$ and thus negligible.

\section{Appendix: Frequency-dependent scaling function $S(z)$}

Bhattacharjee and Ferrell calculated the frequency-dependent viscosity of a classical fluid near its critical point. The relevant portion of their results in Reference [8] are reproduced here, correcting two typographic errors in the original published expressions for $\ln \left(S_{4}\right)$ and for $R(z)$.

The dependence of the viscosity on correlation length $\xi$ and frequency $f$ is

$$
\eta(\xi, f)=\eta(\xi, 0) S^{z_{\eta} /\left(3+z_{\eta}\right)},
$$

where the argument of the scaling function $S(z)$ is the scaled frequency defined by $z \equiv$ $-i \pi f \tau$. Bhattacharjee and Ferrell used the decoupled-mode theory to calculate $S(z)$ to single-loop order. They accurately approximated their result in closed form by an average of calculations in two and four dimensions.

$$
S \simeq\left(\widetilde{S}_{2}\right)^{2 / 3}\left(\widetilde{S}_{4}\right)^{1 / 3}
$$

Here, the tilde refers to additional rescalings given by

$$
\widetilde{S}_{2}(z) \equiv S_{2}\left((2 / e)^{3} z\right) \quad \text { and } \quad \widetilde{S}_{4} \equiv S_{4}((8 / e) z) .
$$

The scaling functions $S_{2}$ and $S_{4}$ are given by

$$
\ln \left(S_{2}\right)=\left(\frac{z}{z-1}\right)+\left(\frac{z}{z-1}\right)^{2} \ln z
$$

and

$$
\ln \left(S_{4}\right)=\left(-3+\frac{2}{z}\right)+\left(1-\frac{3}{z}+\frac{1}{z^{2}}\right) \ln z+\left(5-\frac{5}{z}+\frac{1}{z^{2}}\right) R(z),
$$




\section{References}

[1] P.C. Hohenberg and B.I. Halperin, Rev. Mod. Phys. 49, 435 (1977).

[2] M.R. Moldover, J.V. Sengers, R.W. Gammon, and R.J. Hocken, Rev. Mod. Phys. 51, 79 (1979).

[3] R.W. Gammon, J.N. Shaumeyer, M.E. Briggs, H. Boukari, D. Gent, and R.A. Wilkinson, page 137, Proceedings of the 1997 NASA/JPL Microgravity Fundamental Physics Workshop, (NASA Document D-15677, JPL, Pasadena, CA) (1998).

[4] R.A. Wilkinson, G.A. Zimmerli, H. Hao, M.R. Moldover, R.F. Berg, W.L. Johnson, R.A. Ferrell, and R.W. Gammon, Phys. Rev. E 57, 436 (1998).

[5] G.A. Zimmerli, R.A. Wilkinson, R.A. Ferrell, and M.R. Moldover, preprint (1998).

[6] J. Straub, A. Haupt, and L. Eicher, Int. J. Thermophys. 16, 1033 (1995).

[7] Y. Garrabos, B. Le Neindre, P. Guenoun, B. Khalil, and D. Beysens, Eurphys. Lett. 19, 491 (1992).

[8] J.K. Bhattacharjee and R.A. Ferrell, Phys. Rev. A 27, 1544 (1983).

[9] J.K. Bhattacharjee, R.A. Ferrell, R.S. Basu, and J.V. Sengers, Phys. Rev. A 24, 1469 (1981). (The crossover function from this reference is also summarized in Appendix $A$ of Reference [10].)

[10] R.F. Berg and M.R. Moldover, J. Chem. Phys. 93, 1926 (1990).

[11] L. Bruschi and M. Santini, Phys. Lett. A 73, 395 (1979).

[12] L. Bruschi, Il Nuovo Cimento 1D, 362 (1982). 
[13] Y. Izumi, Y. Miyake, and R. Kono, Phys. Rev. A 23, 272 (1981).

[14] R.F. Berg and M.R. Moldover, J. Chem. Phys. 89, 3694 (1988).

[15] H. Hao, R.A. Ferrell, and J.K. Bhattacharjee, preprint (1997). Hao et al. used the value $\eta=0.040$ for the exponent $\eta$ that appears in the correlation function to obtain $z_{\eta}=0.066$. We used the value $\eta=0.035$ (see $[33,34]$ ) in their expressions to obtain $z_{\eta}=0.067$.

[16] R.A. Ferrell and J.K. Bhattacharjee, Phys. Rev. A 31, 1788 (1985).

[17] R.F. Berg and M.R. Moldover, Science Requirements Document, report to NASA Lewis Research Center, 60009-DOC-006 (1993).

[18] A.M. Peddie, Flight instrument specification for the Critical Viscosity of Xenon flight project, 60009-DOC-014 (1996).

[19] M.J. Assael, Z.A. Gallis, and V. Vosovic, High Temp. - High Press. 28, 583 (1996).

[20] In order to describe materials and experimental procedures adequately, it is occasionally necessary to identify commercial products by manufacturer's name or label. In no instance does such identification imply endorsement by the National Institute of Standards and Technology, nor does it imply that the particular product or equipment is necessarily the best available for the purpose.

[21] R.F. Berg, Rev. Sci. Instrum. 66, 4665 (1995).

[22] R.F. Berg, G.A. Zimmerli, and M.R. Moldover, Int. J. Thermophys. 19, 481 (1998).

[23] D.W. Oxtoby, J. Chem. Phys. 62, 1463 (1975).

[24] I.S. Steinhart and S.R. Hart, Deep Sea Res. 15, 497 (1968).

[25] V.A. Rabinovich, A.A. Vasserman, V.I. Nedostup, L.S. Veksler, Thermophysical Properties of Neon, Argon, Krypton, and Xenon (Hemisphere Publishing, Washington, 1987). 
[26] R.F. Berg, M.J. Lyell, G.B. McFadden, and R.G. Rehm, Phys. Fluids 8, 1464 (1996).

[27] R.F. Berg, Int. J. Thermophys. 16, 1257 (1995).

[28] R.E. Williams and R.G. Hussey, Phys. Fluids 11, 2083 (1972).

[29] U. Närger and D.A. Balzarini, Phys. Rev. B 42, 6651 (1990).

[30] J. Luettmer-Strathmann, J.V. Sengers, and G.A. Olchowy, J. Chem. Phys. 103, 7482 (1995).

[31] J.V. Sengers and J. Luettmer-Strathmann, Chapter 6 in Transport Properties of Fluids, edited by J. Millat, J.H. Dymond, and C.A. Nieto de Castro (Cambridge University Press, New York, 1996).

[32] J.V. Sengers, private communication (1998).

[33] H.W.J. Blöte, E. Luijten, and J.R. Heringa, J. Phys. A: Math. Gen. 28, 6289 (1995).

[34] R. Guida and J. Zinn-Justin, Nucl. Phys. B 489 [FS], 626 (1997).

[35] H. Hao, R.A. Ferrell, and J.K. Bhattacharjee, preprint (1997).

[36] E.D. Siggia, B.I. Halperin, and P.C. Hohenberg, Phys. Rev. B 13, 2110 (1976).

[37] H. Güttinger and D.S. Cannell, Phys. Rev. A 24, 3188 (1981).

[38] J.K. Bhattacharjee and R.A. Ferrell, Phys. Lett. 27A, 290 (1980).

[39] K.R. Spangenberg, Vacuum Tubes (McGraw-Hill, New York, 1948).

[40] J.V. Sengers and M.R. Moldover, Phys. Lett. 66A, 44 (1978).

[41] R. Tofeu, B. Le Neindre, and P. Bury, Compte Rendus Acad. Sci. Paris B 273, 113 (1971). 


\section{E. Figures}

1. Log-log plot of xenon's viscosity measured near the critical point. The asymptotic line has the slope $z_{\eta} / \nu=0.0435$ deduced from the present microgravity data. Near $T_{c}$, the CVX microgravity data $(\operatorname{Re}(\eta)$ at $2 \mathrm{~Hz})$ depart from the asymptotic line because of viscoelasticity. The two sets of ground data depart from the asymptotic line further from $T_{c}$ because the xenon stratified in Earth's gravity.

2. Xenon's viscosity at critical density measured at frequencies from 2 to $12 \mathrm{~Hz}$. The solid curves resulted from fitting Eq.(1.2) to the data in the range $10^{-6}<t<10^{-4}$. (a) The real viscosity $\operatorname{Re}(\eta)$. Near $t=10^{-5}$, the data depart from the $0 \mathrm{~Hz}$ curve because of viscoelasticity. (b) The ratio $\operatorname{Im}(\eta) / \operatorname{Re}(\eta)$. For clarity, the ratio data at frequencies above $2 \mathrm{~Hz}$ are displaced downward by integer multiples of 0.005 ; otherwise they would coincide at $t>10^{-5}$.

3. The temperature timeline followed by CVX. Both panels show the same data but with different vertical scales.

4. Viscometer response as a function of temperature very near $T_{c}$. Both panels show data at three ramp rates. On the lower panel, the temperature scale is expanded by a factor of 8. (a) Above $T_{c}$, data collected at the fastest ramp rate of $-33 \mu \mathrm{K} \cdot \mathrm{s}^{-1}$ reflect the $0.3 \%$ lower density caused by starting the ramp at $T_{c}+0.2 \mathrm{~K}$. (b) Above $T_{c}$, data collected while ramping at $-0.05 \mu \mathrm{K} \cdot \mathrm{s}^{-1}$ agree with those collected five days earlier while ramping 20 times faster.

5. Cutaway view of the CVX viscometer cell. The cylindrical volume occupied by the xenon was $38 \mathrm{~mm}$ long and $19 \mathrm{~mm}$ in diameter. Torque was applied to the screen by applying different voltages to diagonal pairs of electrodes while maintaining the screen at ground potential. 
6. The magnitude of the oscillator's transfer function measured in vacuum and in xenon at critical density.

7. Cross-section of the Experimental cannister.

8. Simplified diagram of the circuit used to drive and detect the oscillator's motion.

9. The normalized density deviations calculated for a timeline similar to that used by CVX. Each curve was calculated for at a radius $r$ within a cylinder with isothermal walls at radius $R_{\text {cell }}$.

10. The oscillator's transfer function $G_{\text {meas }}(f)$ in the frequency region used to determine the viscosity.

11. Oscillations and spikes in the magnitude data at $1 \mathrm{~Hz}$. The oscillation's 45-minute period was half that of the Shuttle's orbital period. The minima of the oscillations occurred when the Shuttle was near the Earth's equator. The three spikes indicated by arrows occurred when the Shuttle was near the South Atlantic Anomaly.

12. Examples of the transfer function's magnitude measured at very low frequencies, where noise was much greater in orbit than on Earth.

13. The calibration function $B(R / \delta)$. The smooth curves indicate $B(R / \delta)$ calculated from the hydrodynamic theory of a transversely oscillating circular cylinder of radius $R=13.4 \mu \mathrm{m}$. (a) Magnitude scaled to reveal departures from the dominant $(R / \delta)^{3 / 2}$ behavior. (b) Phase.

14. The time dependence of the oscillator parameter $k_{t r}$. The solid curve is a fit to the transfer function's magnitude at $0.5 \mathrm{~Hz}$, assuming that $k_{\text {tr }}$ was a linear function of the cell's temperature, the Experiment cannister's temperature, and time.

15. The transfer function's phase at $2 \mathrm{~Hz}$ as a function of time. The height of the boxes corresponds to $0.2 \%$ of the density. The reproducibility of the oscillator's phase at 300 
$\mathrm{mK}, 100 \mathrm{mK}, 30 \mathrm{mK}$, and $3 \mathrm{mK}$ above $T_{c}$ demonstrates that density deviations could be reduced to within $0.1 \%$ of the average density at these temperatures. Filled points were obtained below $T_{c}$. The points denoted "A" and "B" were obtained shortly after warming the sample from below $T_{c}$. As discussed in the text, the equilibration of set $B$ was much slower than that of set $A$.

16. Cusps in the data very close to $T_{c}$. These data were taken at $5 \mathrm{~Hz}$; similar features occurred at other frequencies above $2 \mathrm{~Hz}$. (a) Maximum damping (minimum amplitude) of the oscillator occurred within $0.01 \mathrm{mK}$ of $T_{c}$, corresponding to $t= \pm 3 \times 10^{-7}$. (b) A maximum of the phase located within $20 \mu \mathrm{K}$ of $T_{c}$. The inset shows that the maximum was superposed on a broader minimum.

17. Model cross section of the CVX electrodes used to calculate electric fields. The field near the screen depended on the potentials $V_{1}$ and $V_{2}$, the distances $x_{1}, x_{2}$, and $a$, and the wire radius $R$. 


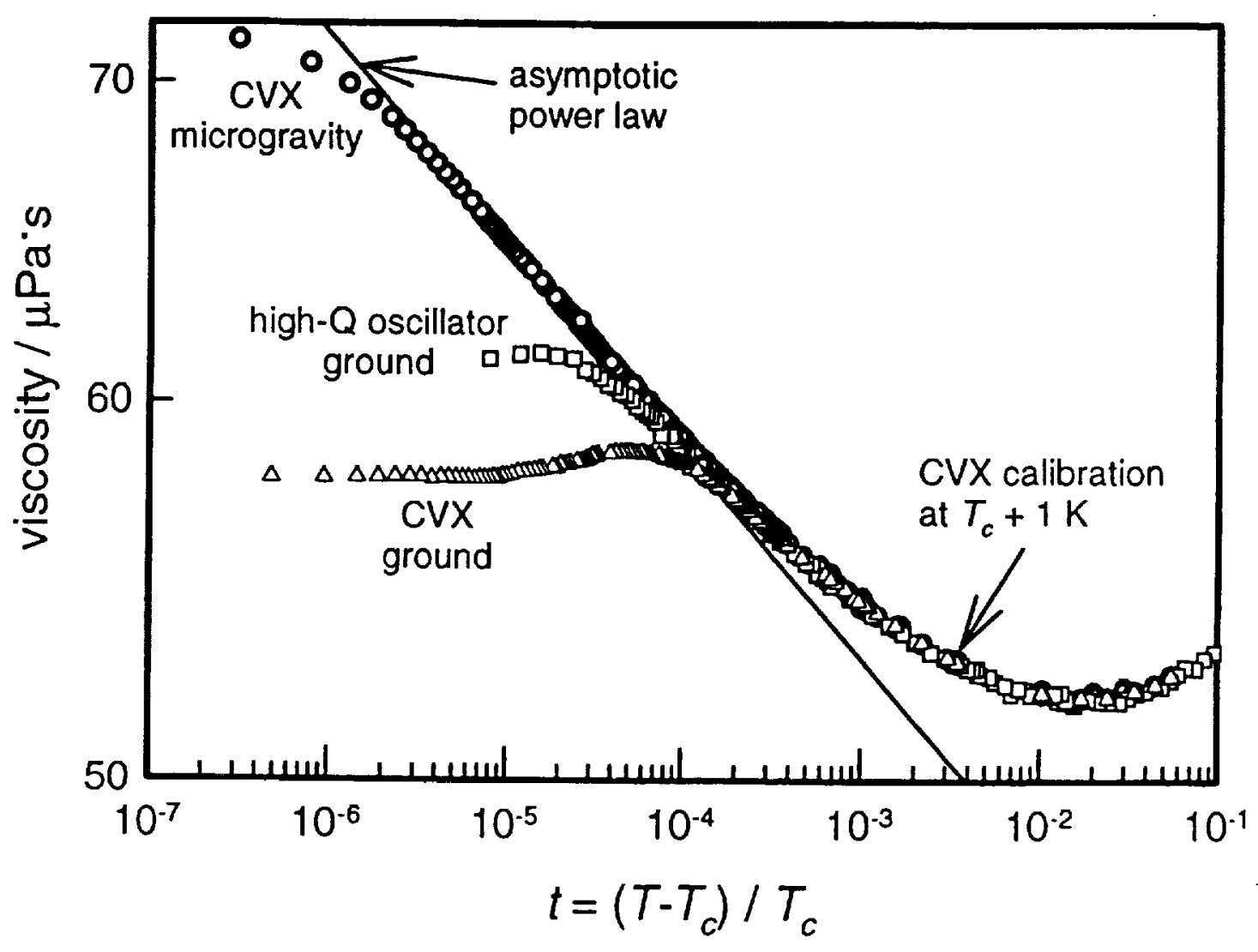




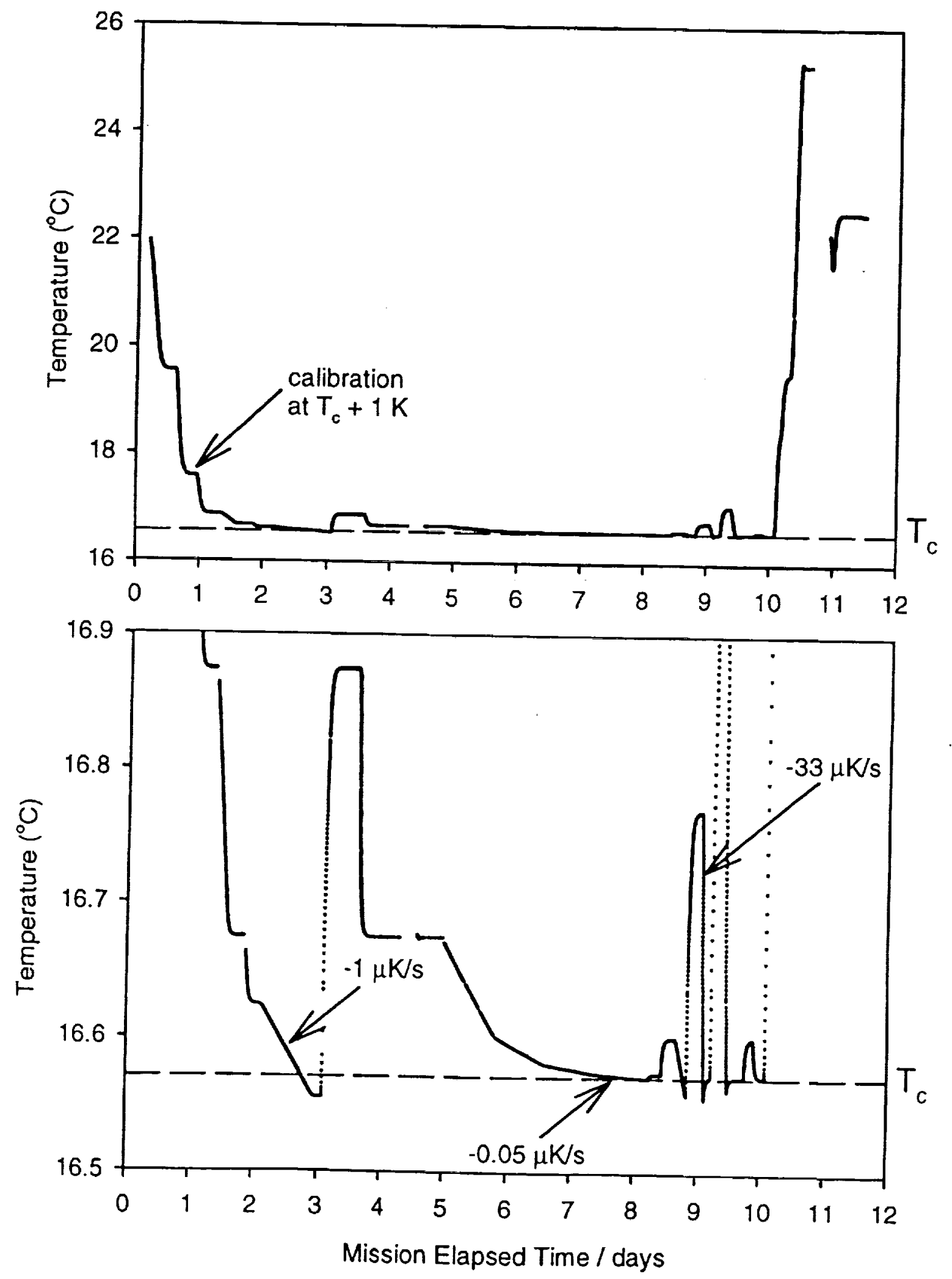



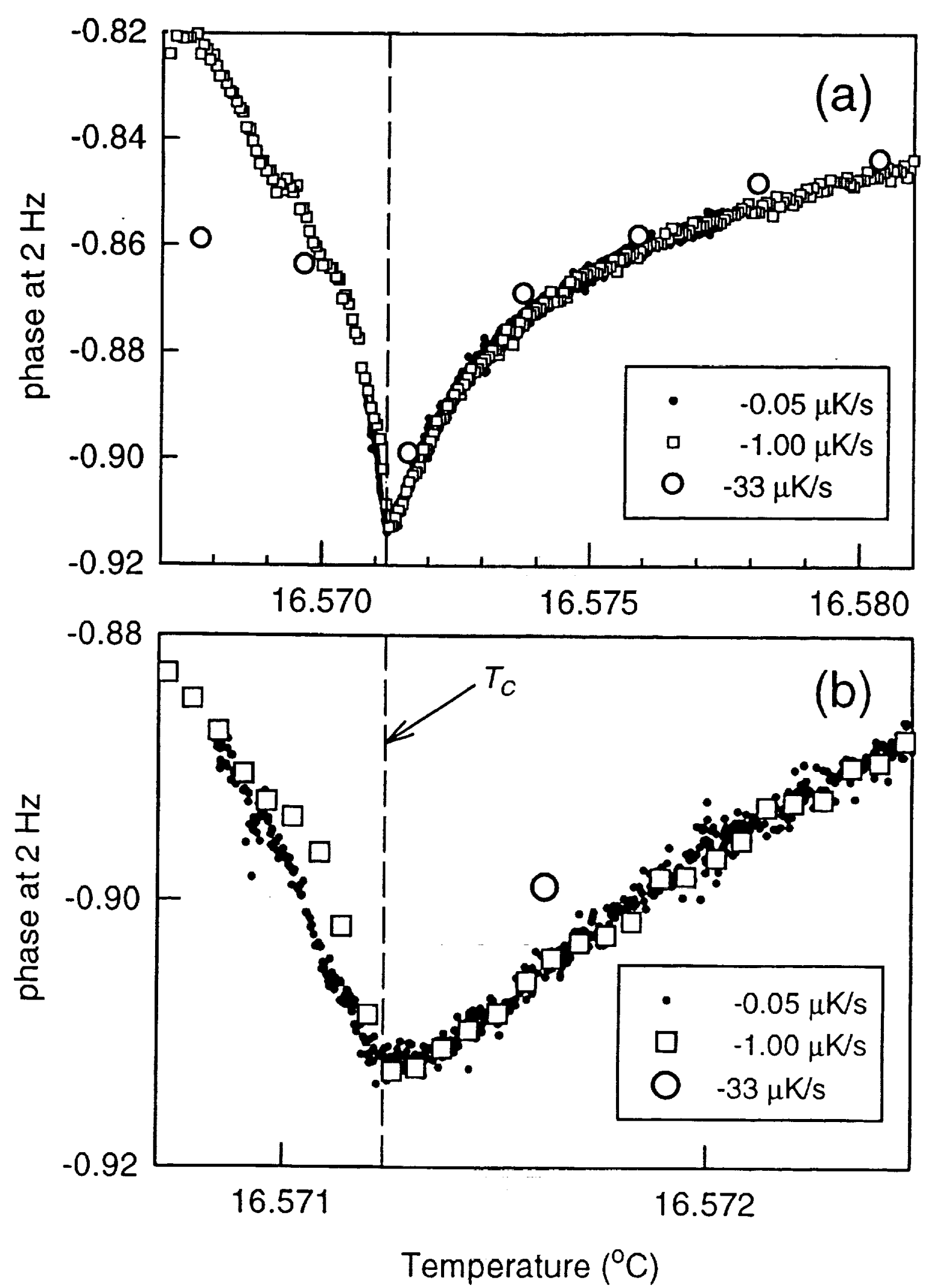


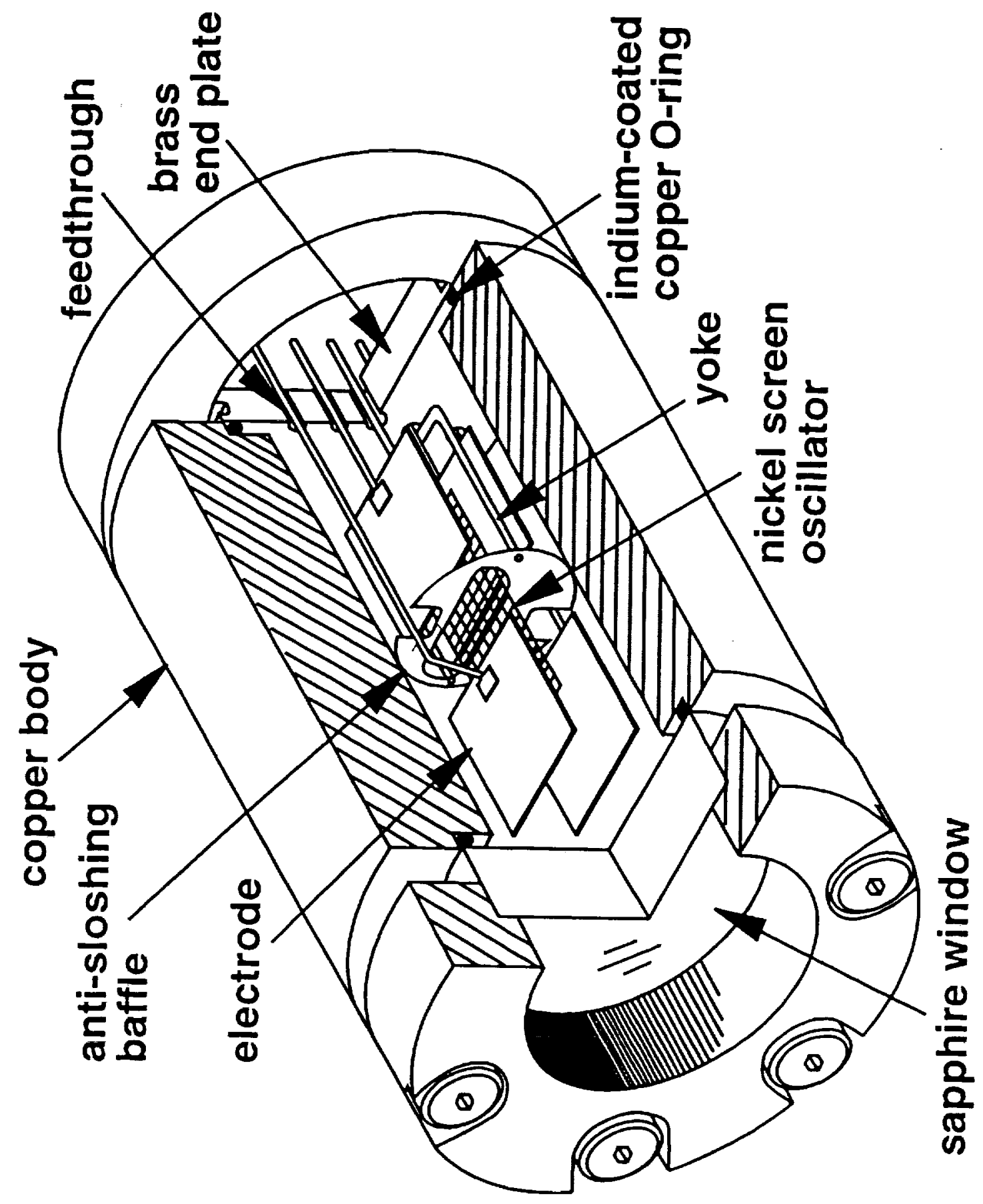




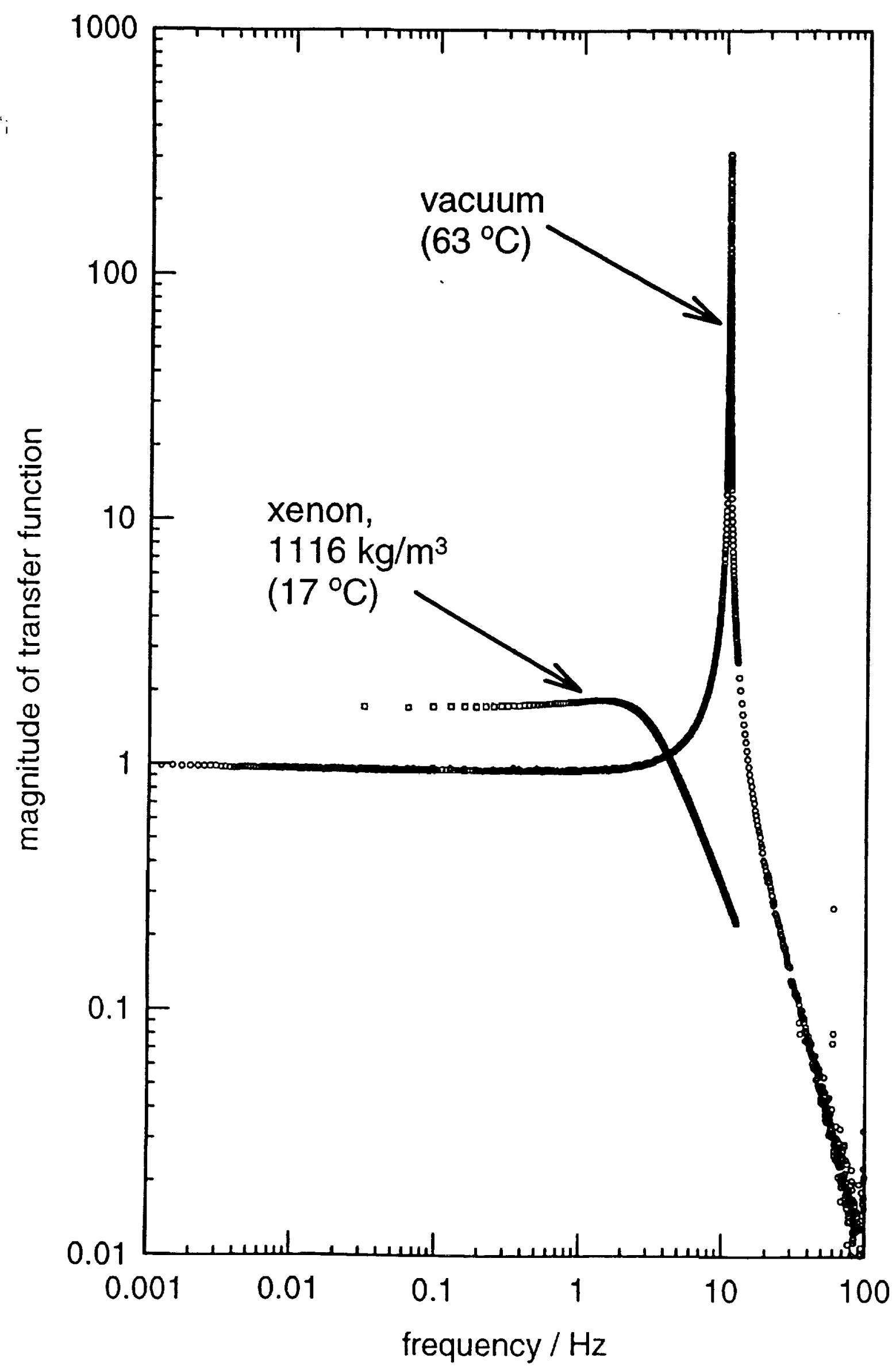




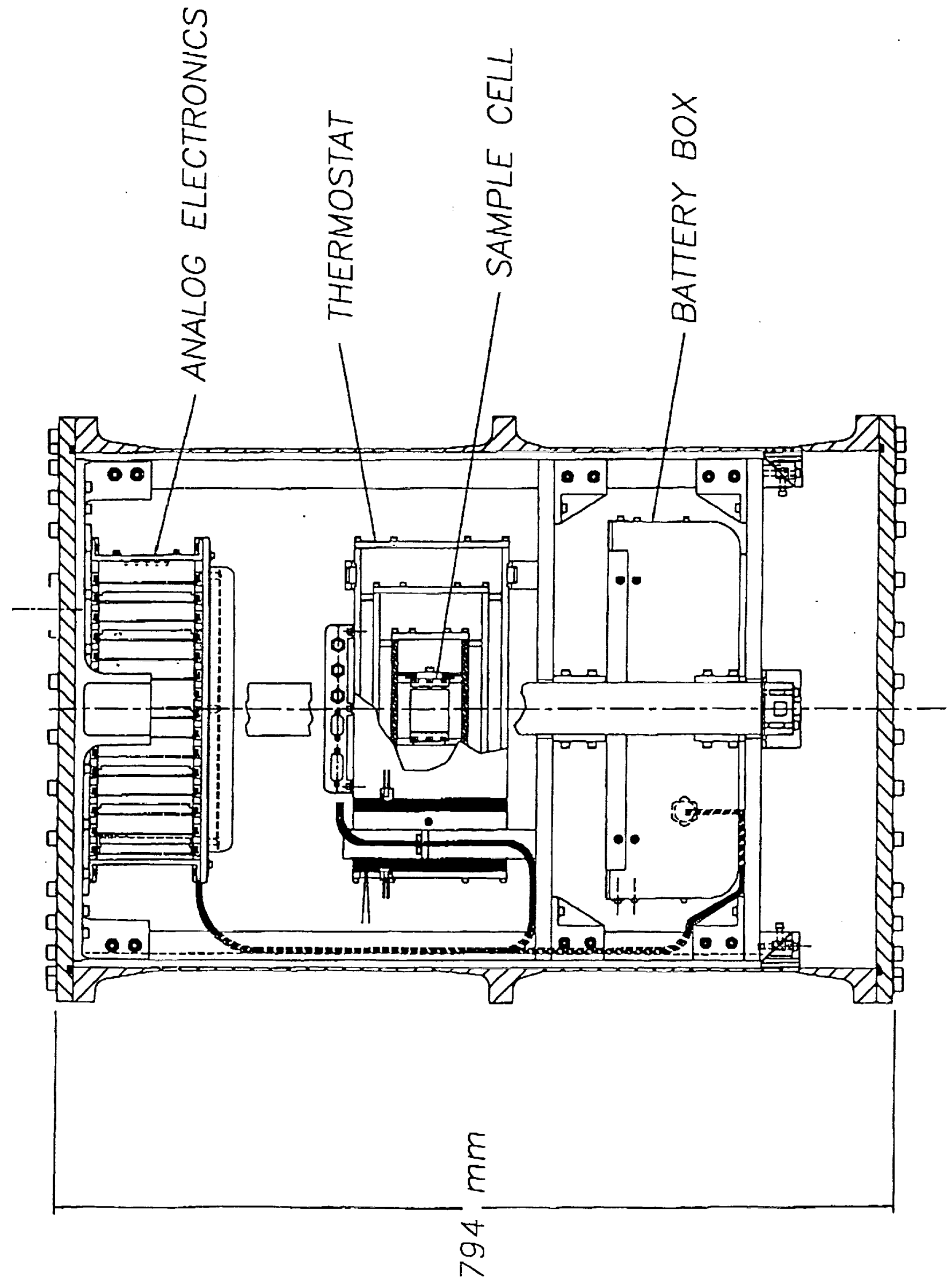




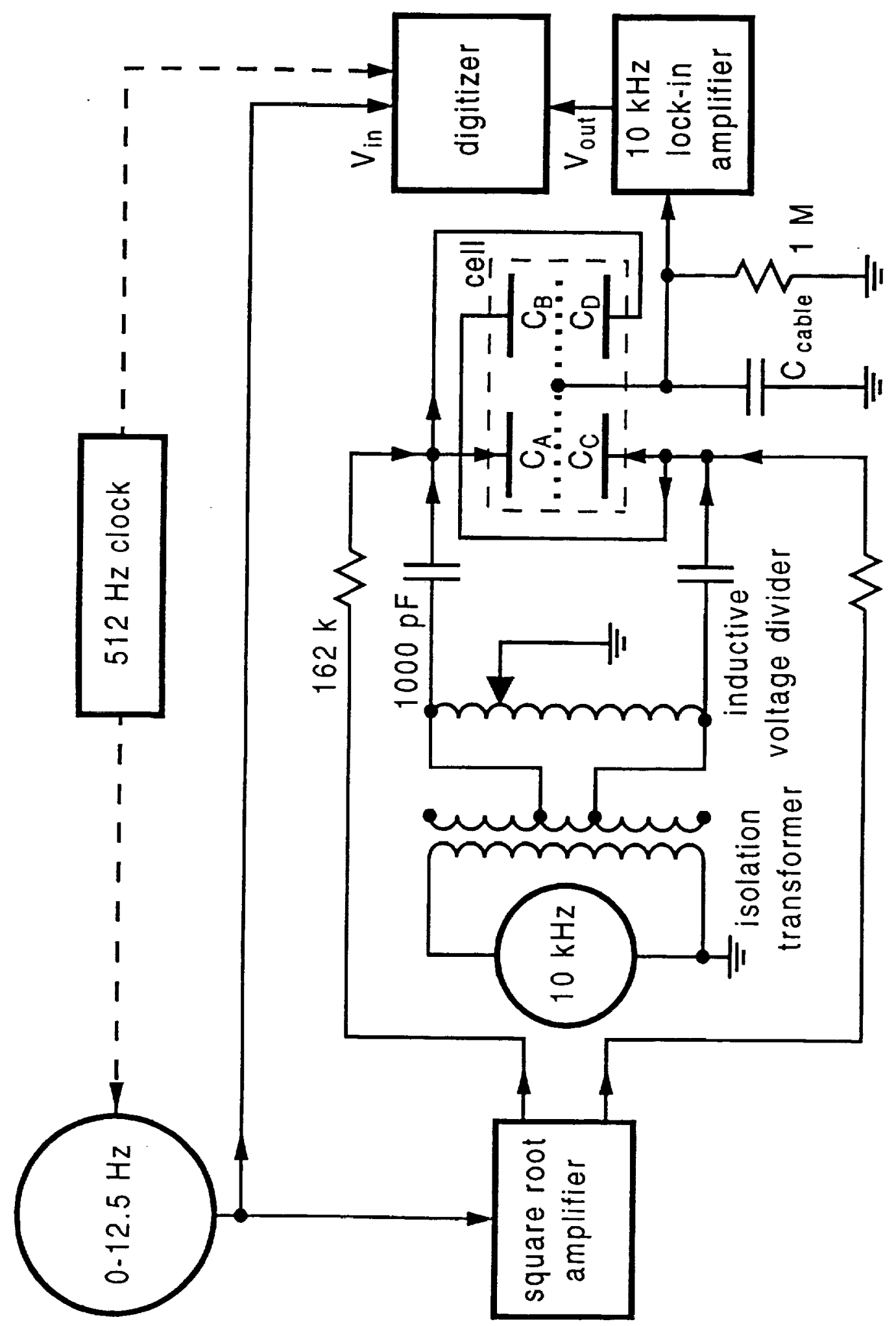




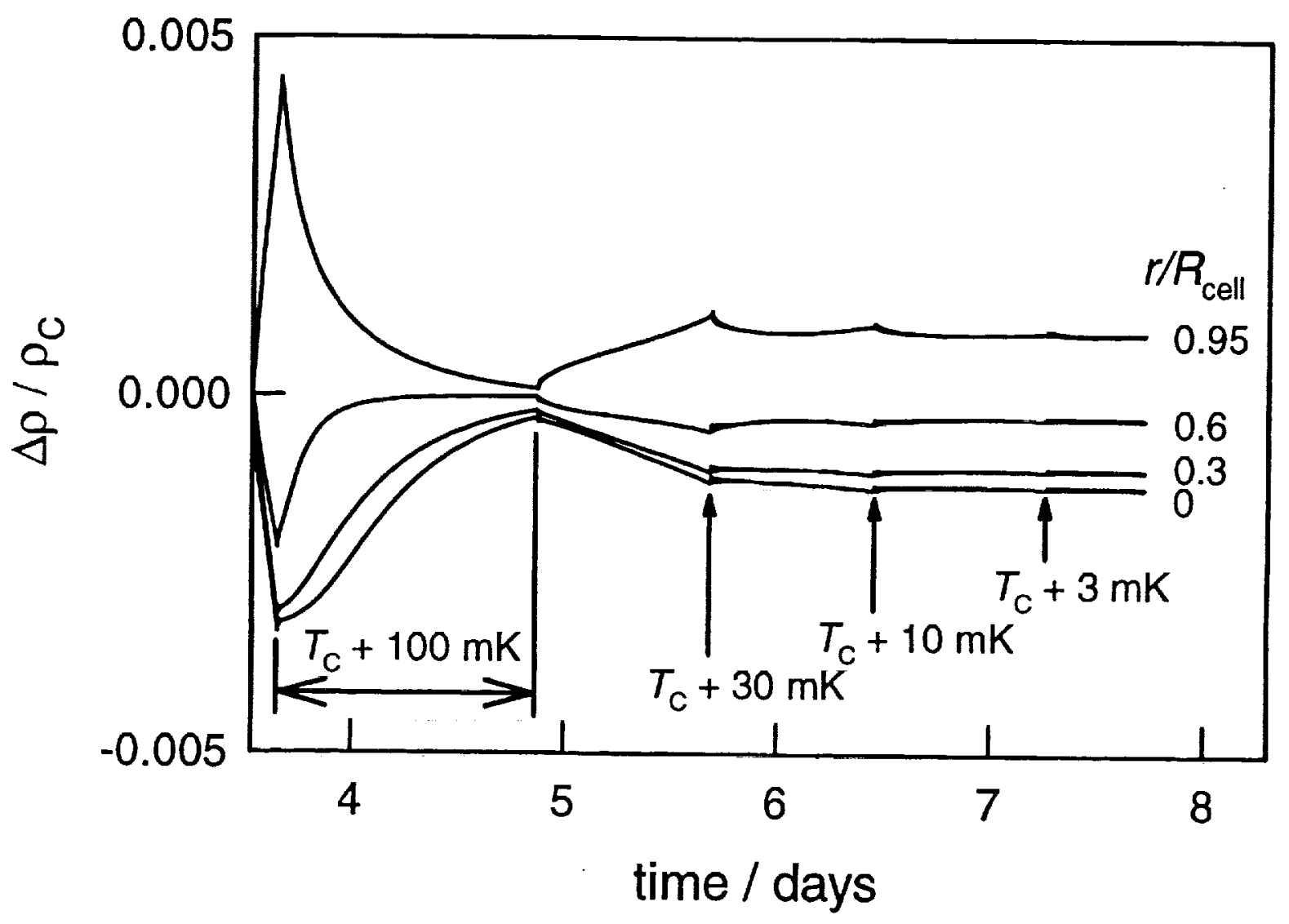




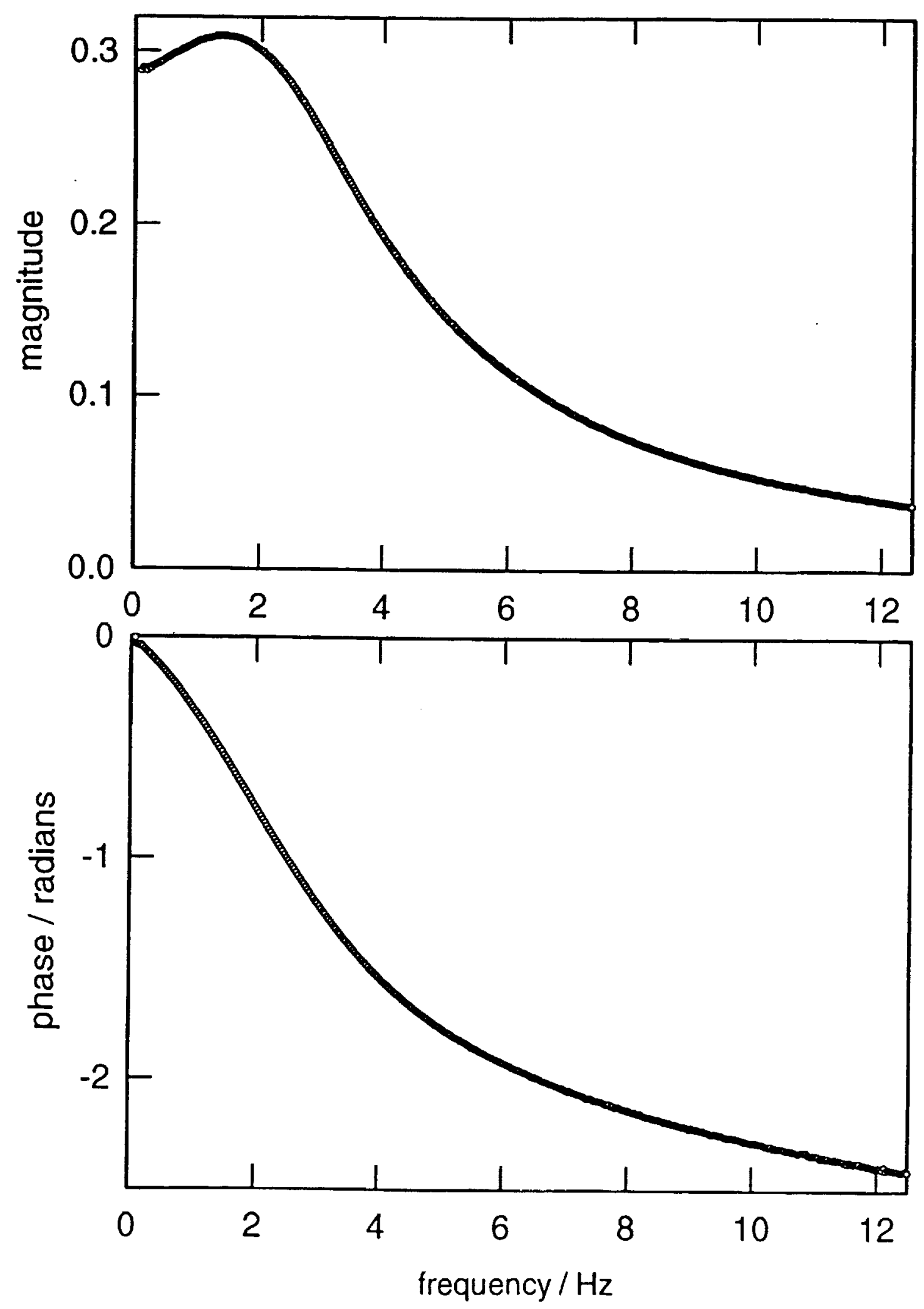




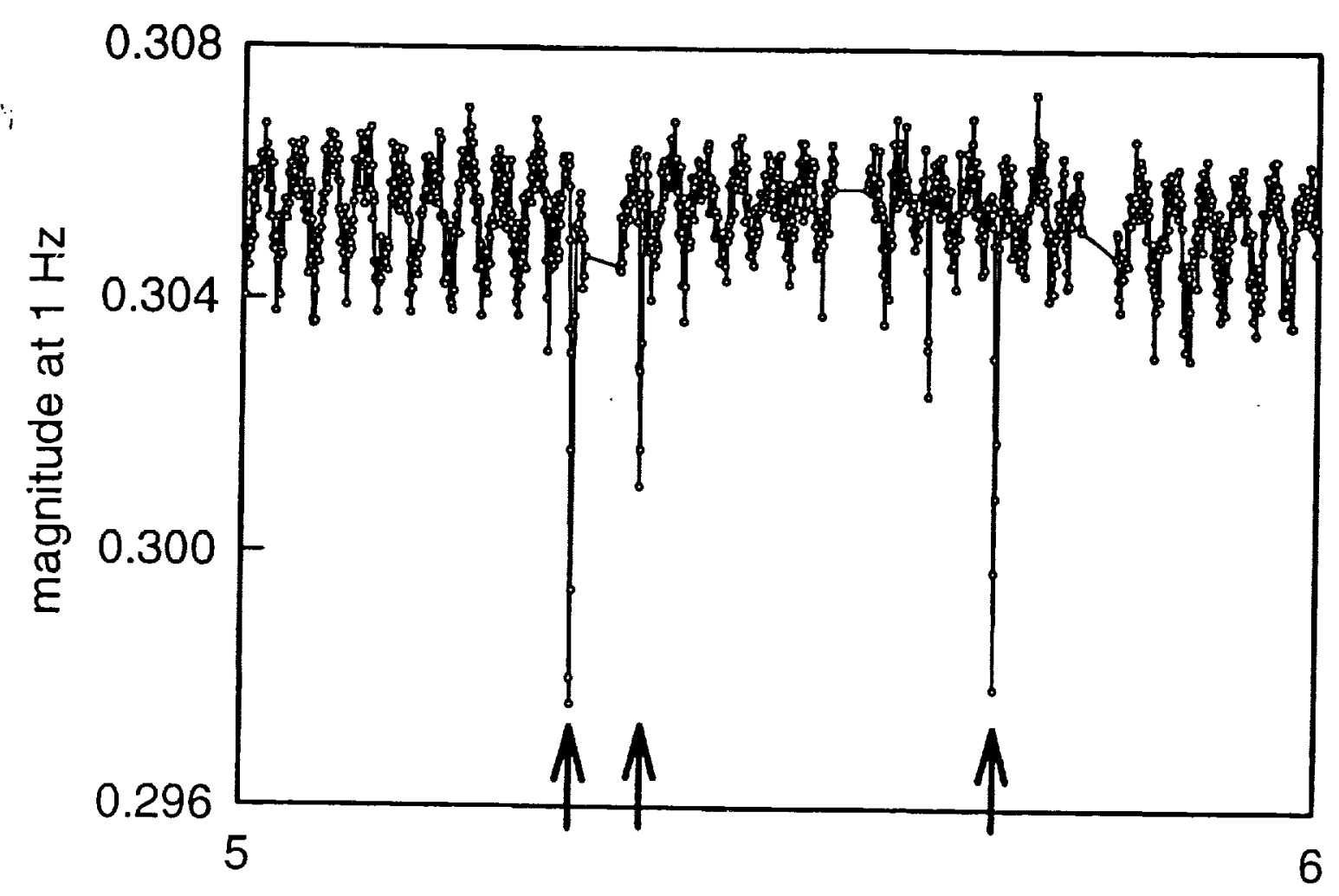

Mission Elapsed Time / days 


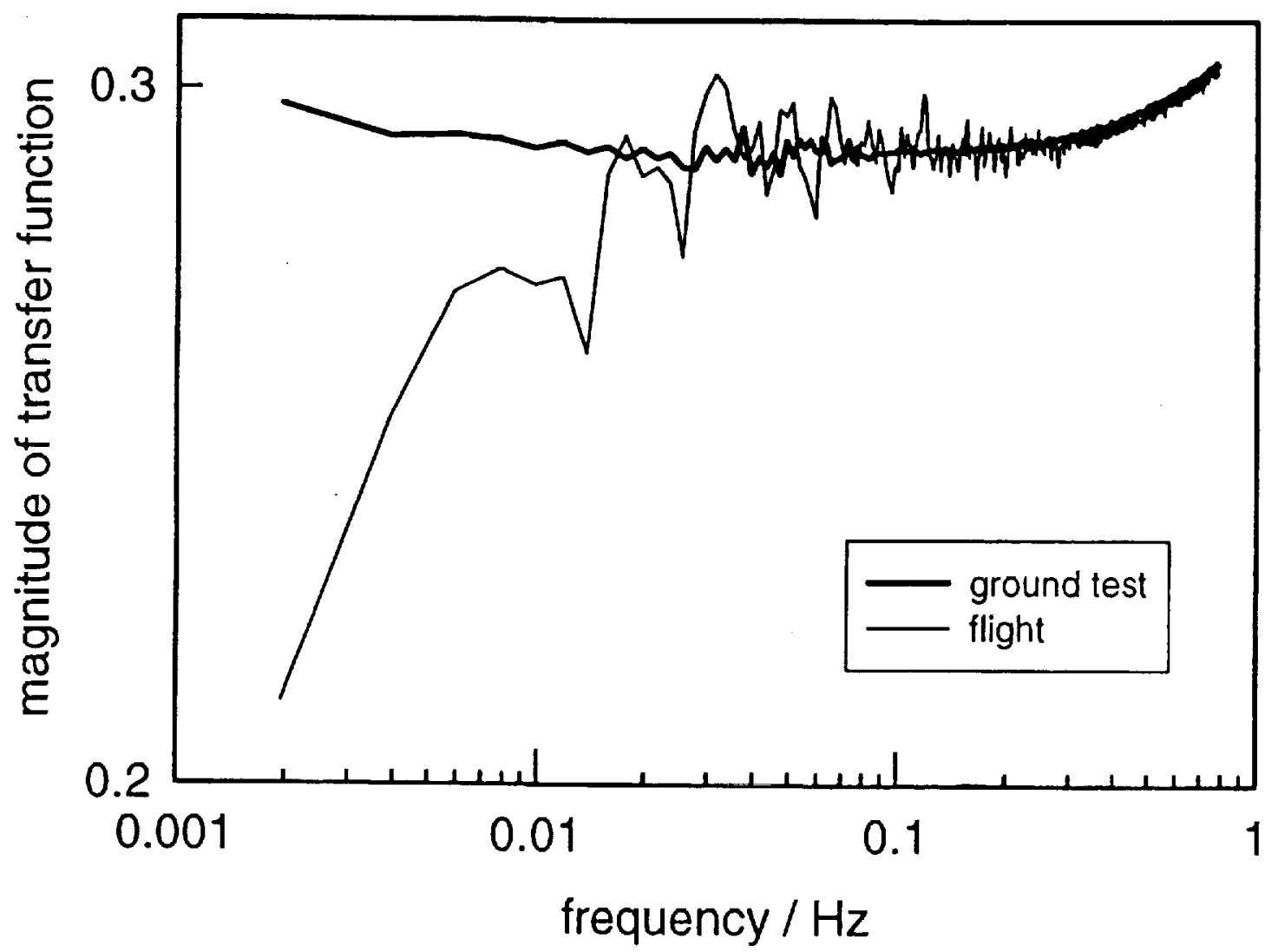



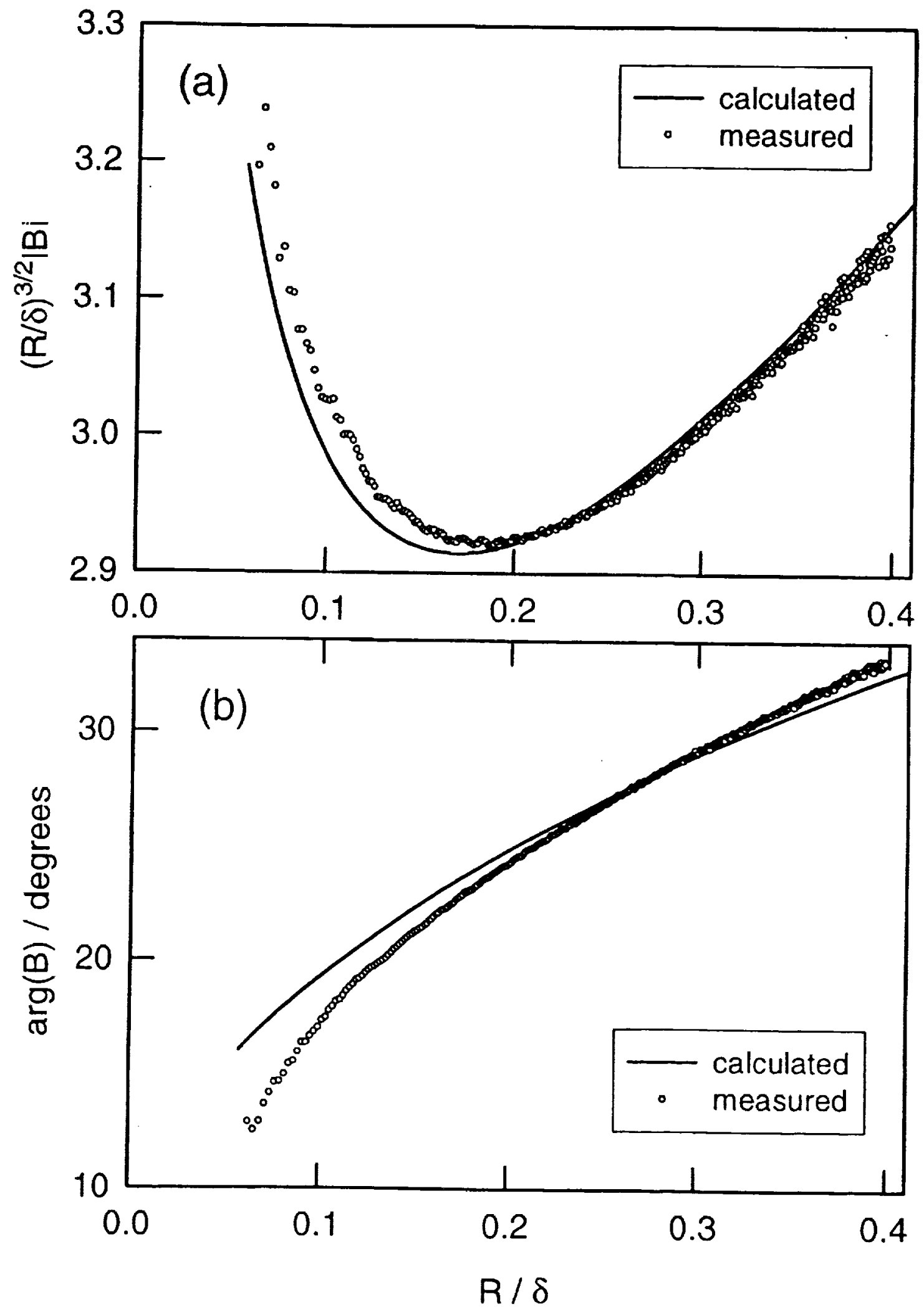


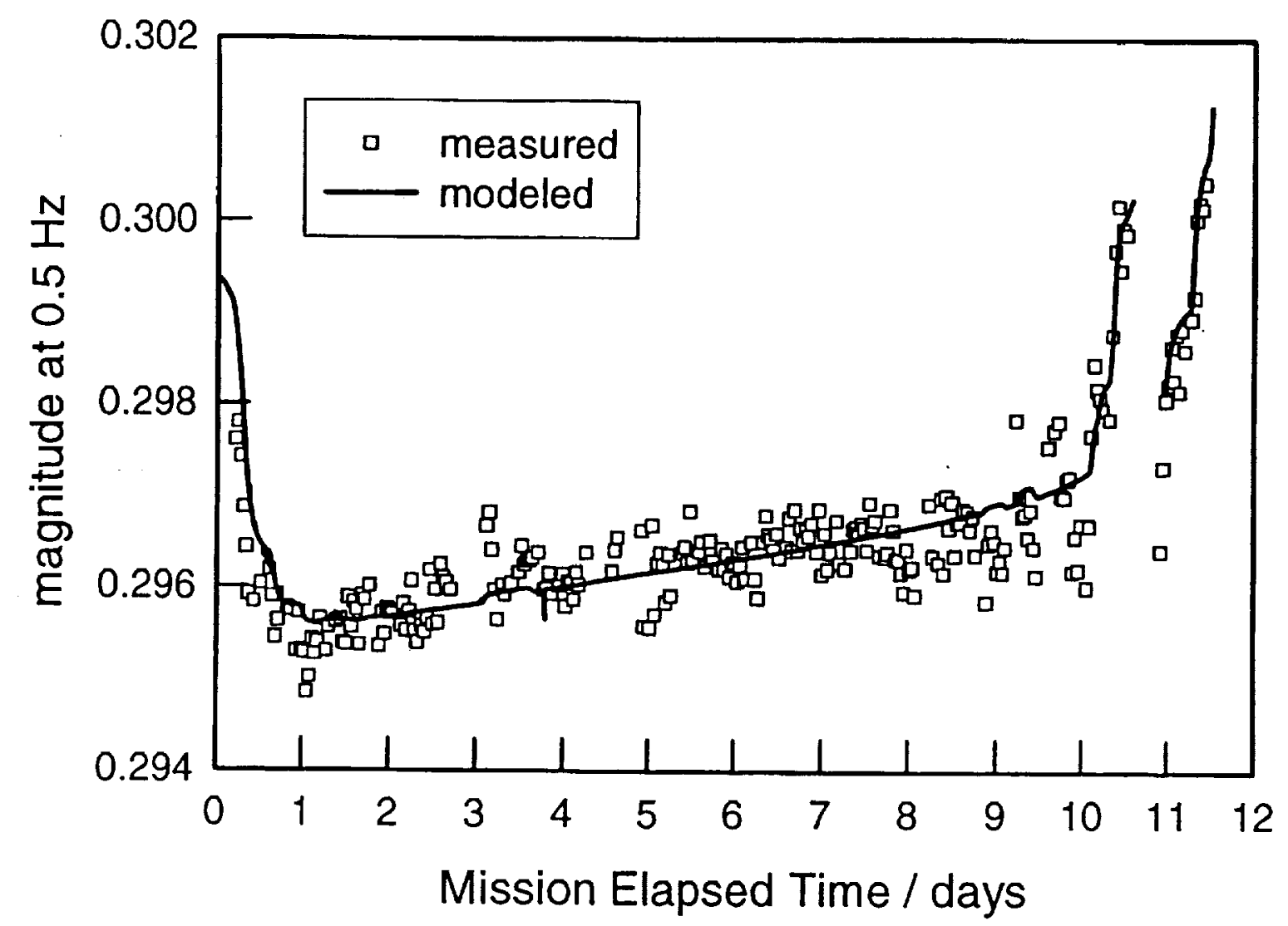




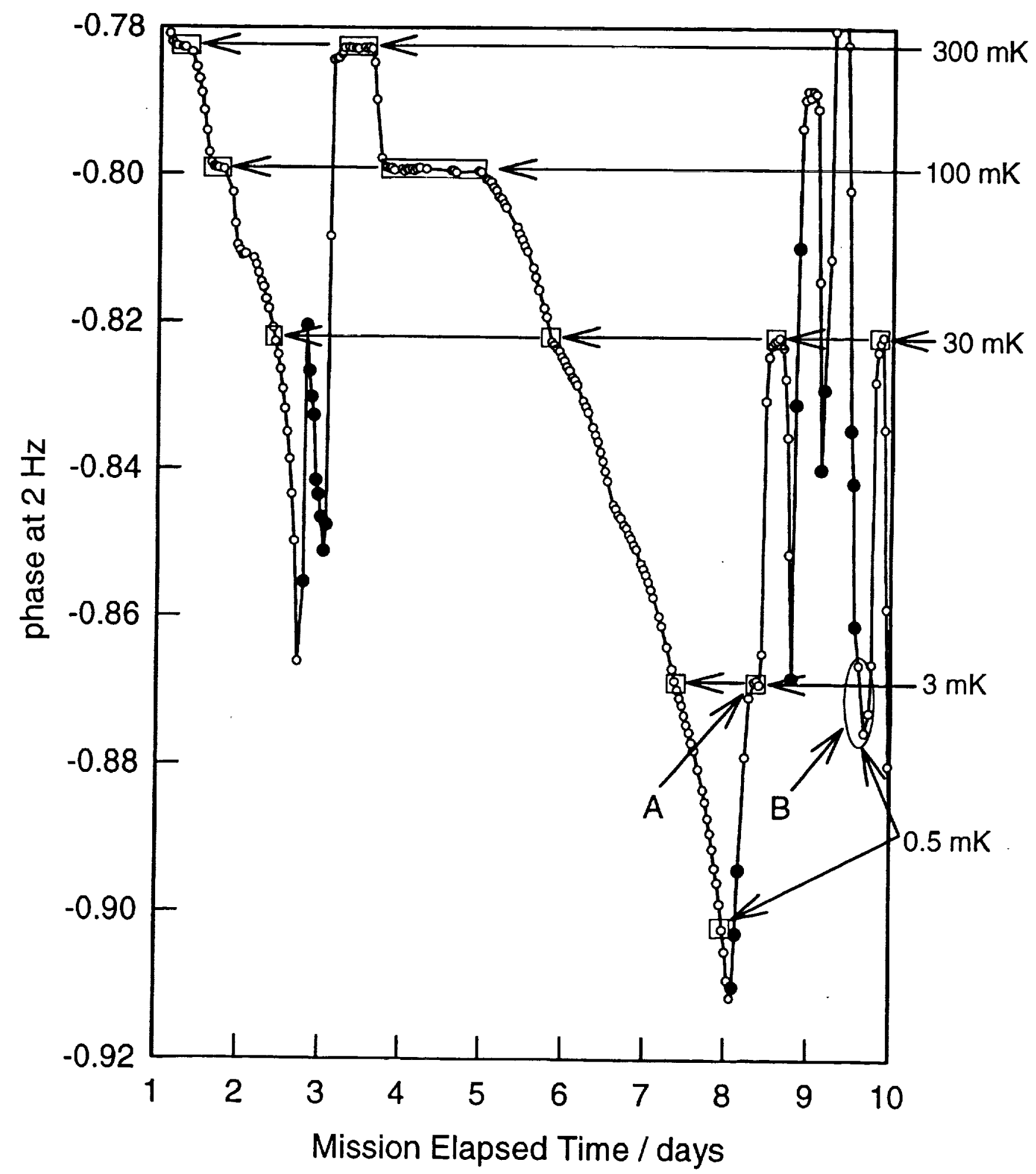

15 

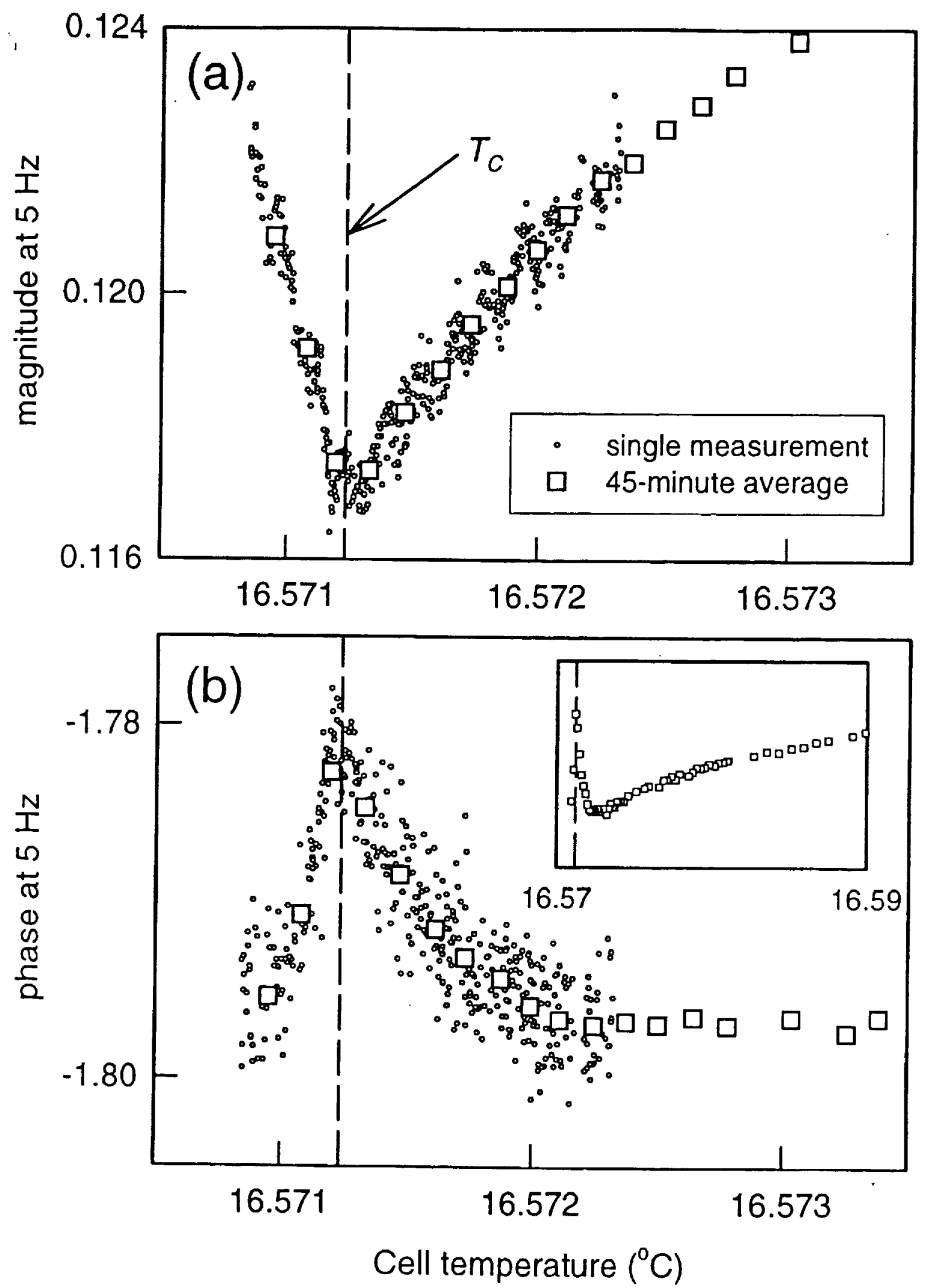


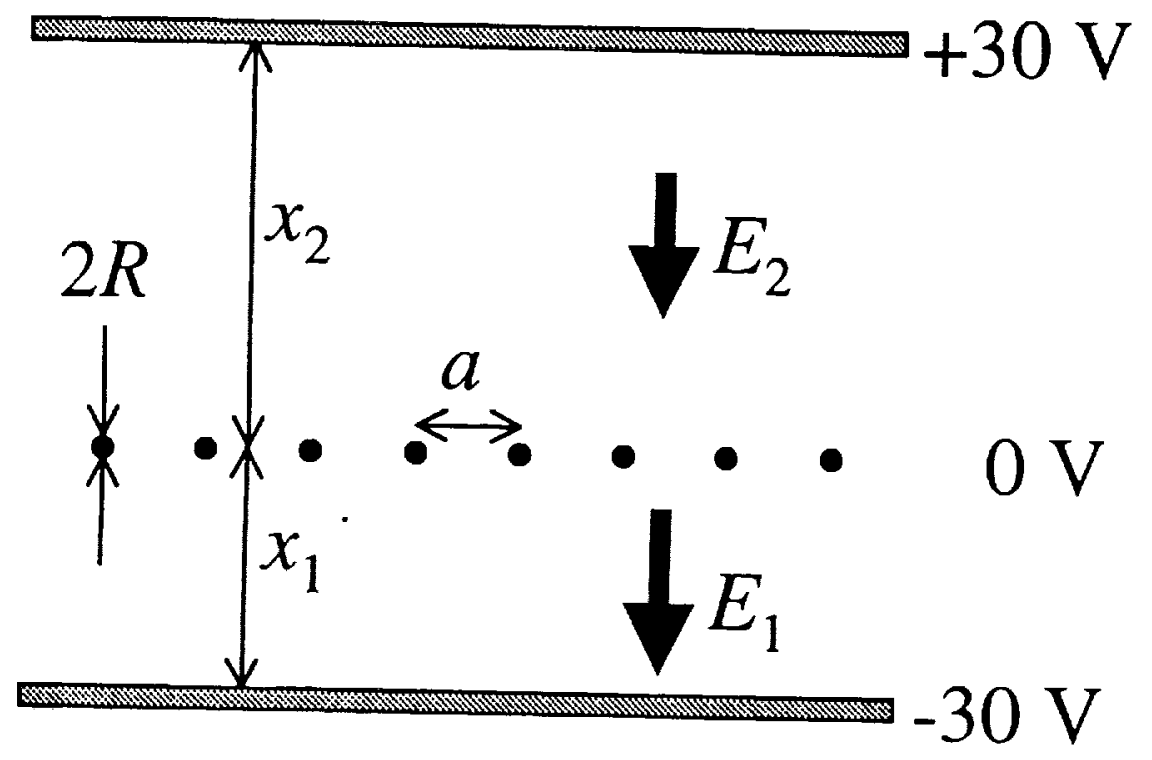

